\title{
DIRECTIONAL INPUT ADAPTATION IN PARAMETRIC OPTIMAL CONTROL PROBLEMS*
}

\author{
SAURABH A. DESHPANDE ${ }^{\dagger}$, DOMINIQUE BONVIN $^{\dagger}$, AND BENOÎT CHACHUAT ${ }^{\ddagger}$
}

\begin{abstract}
This paper deals with input adaptation in dynamic processes in order to guarantee feasible and optimal operation despite the presence of uncertainty. For those optimal control problems having terminal and mixed control-state path constraints, two orthogonal sets of adaptation directions can be distinguished in the input space: the sensitivity-seeking directions, along which a small variation from an optimal nominal solution will not affect the respective active constraints, and the complementary constraint-seeking directions, along which a variation will affect the respective constraints. It follows that selective input adaptation strategies can be defined, namely, adaptation in the sensitivity- and constraint-seeking directions. This paper proves the important result that, for small parametric perturbations, the cost variation resulting from adaptation in the sensitivity-seeking directions (over no input adaptation) is typically smaller than the cost variation due to adaptation in the constraint-seeking directions. It is also established that no selective input adaptation along a sensitivity-seeking direction can reduce the dominant, first-order term in the optimality gap; adaptation along a constraint-seeking direction is necessary to cancel it out. These results are illustrated with two numerical case studies.
\end{abstract}

Key words. parametric optimal control problem, terminal constraints, mixed control-state constraints, sensitivity-seeking directions, constraint-seeking directions, linear integral equations, selective input adaptation, cost variation, optimality loss

AMS subject classifications. 49K15, 49K40, 49M05, 49M25

DOI. $10.1137 / 110820646$

1. Introduction. A large class of industrial processes is transient in nature. For instance, many processes in the resource industries are either discontinuous or operated in an unsteady-state manner. Batch and semibatch processes in the chemical process industry are other examples of processes that are characterized by the absence of a steady state. Many alternative technologies in the energy sector also exhibit discontinuous operation, and transient energy-generation systems are expected to play a key role in years to come.

In engineering applications, the optimization of transient processes aims at computing time profiles of certain process variables to improve process performancemeasured in terms of profit or product quality - subject to tight operational constraints, including strict safety requirements and environmental regulations. Many numerical methods have been developed over the years to solve such optimal control problems on the basis of a process model; see, e.g., $[3,4,16]$. However, in applying the computed optimal profiles open-loop to the process, both plant-model mismatch and process disturbances typically result in suboptimal process operation or, which is worse, infeasible operation.

A common practice of dealing with uncertainty is to represent it in the form of parametric perturbations. The optimal input profiles are computed off-line for the

${ }^{*}$ Received by the editors January 11, 2011; accepted for publication (in revised form) April 16, 2012; published electronically July 26, 2012. This paper is based upon work supported by the Swiss National Science Foundation (SNSF) under grant 200020-113292.

http://www.siam.org/journals/sicon/50-4/82064.html

${ }^{\dagger}$ Laboratoire d'Automatique, École Polytechnique Fédérale de Lausanne (EPFL), Station 9, CH1015 Lausanne, Switzerland (saurabh.deshpande@epfl.ch, dominique.bonvin@epfl.ch).

${ }^{\ddagger}$ Corresponding author. Centre for Process Systems Engineering (CPSE), Department of Chemical Engineering, Imperial College London, London SW7 2AZ, UK (b.chachuat@imperial.ac.uk). 
nominal values of the parameters. Naturally, when some parameters deviate from their nominal values, a change in optimal inputs is required to maintain optimality and meet constraints. Ideally, one needs to repeat the computations with the modified values of the parameters to obtain the modified optimal inputs $[12,36]$. Efficient approaches that minimize the computational burden have been developed, many of which rely on fast sensitivity updates $[11,23,37,36]$. In another class of approaches, one tries to avoid resolving the optimal control problem by adapting the nominal optimal inputs, thereby reducing the optimality gap [6, 28, 24, 19, 18]. Here again, approximations of the perturbed optimal solutions can be obtained via sensitivity analysis. In particular, extensive work has been done regarding the sensitivity analysis of parametric optimal control problems over the last decades, including the differentiability of the optimal inputs with respect to parameter values [21, 29, 26, 28, 22]. Other ways of approximating the solution of parametric constrained optimization problems include spectral finite-element methods [1].

The main focus of this paper is on selective input adaptation in nonlinear control problems. This is motivated by the observation that adapting all parts of the optimal input profiles to compensate for the effect of parametric perturbations is rarely possible in practice, nor is it expedient from a performance viewpoint. Partial or selective input adaptation scenarios that result in suboptimal process operation with acceptable performance loss hold much promise for improving the computational efficiency of real-time optimization schemes and are therefore worth analyzing.

This paper builds upon and extends previous work on selective adaptation of optimization problems governed by stationary processes [15, 9], a brief overview of which is presented in the appendix; see also [7]. The analysis begins with the identification of selective input adaptation directions around the nominal optimal solution. Specifically, the space of input variations is split into two orthogonal subsets of directions, namely the sensitivity- and constraint-seeking subspaces. This splitting is such that an input variation along any of the sensitivity-seeking directions will not affect the active terminal and path constraints, whereas an input variation along a constraintseeking direction will. In dynamic processes, these directions must be defined at every time $t$ within the optimization horizon, with the extra challenge that all past input variations up to $t$, not merely the input variations at $t$, must be taken into account.

Characterization of these subspaces allows one to analyze the effect of two different selective input adaptation strategies in the presence of parametric perturbations. The effect of either strategy can be quantified in terms of cost variation by comparing its performance with the case of no adaptation. An important contribution of this paper will be to establish that, for small parametric perturbations, the cost variation resulting from input adaptation along the sensitivity-seeking directions is typically smaller than that resulting from adaptation along the constraint-seeking directions. It will also be established that no selective input adaptation along a sensitivity-seeking direction can reduce the dominant (first-order) term in the optimality gap; only an adaptation along a constraint-seeking direction can cancel it out.

The outline of the paper is as follows. The general mathematical formulation and assumptions regarding the parametric optimal control problem are given in section 2. The sensitivity- and constraint-seeking directions are defined in section 3, along with the concept of selective input adaptation. Section 4 presents a quantitative comparison of the cost variation obtained with either adaptation strategy and analyzes the resulting optimality gap. The results of sections 3 and 4 are demonstrated using two case studies in section 5 , based on a tailored numerical procedure that computes 
the sensitivity- and constraint-seeking components of a given input variation. Finally, section 6 summarizes the results and discusses possible extensions to more general optimal control problems.

2. Preliminaries. In this section, the general mathematical formulation for the parametric optimal control problem involving both terminal and mixed control-state path constraints is given, along with a summary of the necessary conditions of optimality (NCOs) and the corresponding assumptions. The terminology of switching times of mixed control-state constraints and related notation are also defined.

2.1. Problem formulation and assumptions. The following parametric optimal control problem $\mathrm{OC}(\boldsymbol{\theta})$, subject to terminal and mixed control-state inequality constraints, with given initial time $t_{0}$ and terminal time $t_{\mathrm{f}}$, is considered:

$$
\begin{array}{cl}
\min _{\mathbf{u}, \mathbf{p}} & J(\mathbf{u}, \mathbf{p})=\varphi\left(\mathbf{x}\left(t_{\mathrm{f}}\right), \mathbf{p}, \boldsymbol{\theta}\right)+\int_{t_{0}}^{t_{\mathrm{f}}} \ell(t, \mathbf{x}(t), \mathbf{u}(t), \mathbf{p}, \boldsymbol{\theta}) d t \\
\text { s.t. } & \dot{\mathbf{x}}(t)=\mathbf{f}(t, \mathbf{x}(t), \mathbf{u}(t), \mathbf{p}, \boldsymbol{\theta}) ; \quad \mathbf{x}\left(t_{0}\right)=\mathbf{h}(\mathbf{p}, \boldsymbol{\theta}), \\
& T_{i}\left(\mathbf{x}\left(t_{\mathrm{f}}\right), \mathbf{p}, \boldsymbol{\theta}\right) \leq 0, \quad i=1, \ldots, n_{\mathbf{T}}, \\
& \Omega_{i}(t, \mathbf{x}(t), \mathbf{u}(t), \mathbf{p}, \boldsymbol{\theta}) \leq 0, \quad i=1, \ldots, n_{\boldsymbol{\Omega}},
\end{array}
$$

where $t \in\left[t_{0}, t_{\mathrm{f}}\right], \mathbf{u}(t) \in \mathbb{R}^{n_{\mathbf{u}}}, \mathbf{p} \in \mathbb{R}^{n_{\mathbf{p}}}, \mathbf{x}(t) \in \mathbb{R}^{n_{\mathbf{x}}}$, and $\boldsymbol{\theta} \in \Theta$, with $\Theta$ a subset of $\mathbb{R}^{n_{\boldsymbol{\theta}}}$. Moreover, the functions $\mathbf{f}, \mathbf{h}, \mathbf{T}, \boldsymbol{\Omega}, \boldsymbol{\varphi}$, and $\ell$ are assumed to be continuously differentiable with respect to all of their arguments.

Let the nominal values of the system parameters be $\boldsymbol{\theta}^{0}$, and let $\left(\mathbf{u}^{*}(t), \mathbf{p}^{*}\right)$ be an optimal solution for the problem $\mathrm{OC}\left(\boldsymbol{\theta}^{0}\right)$, with associated optimal response $\mathbf{x}^{*}(t)$. We assume that the active terminal and path constraints satisfy a regularity condition at $\left(\mathbf{u}^{*}(t), \mathbf{p}^{*}\right)$ (constraint qualification) [28]. Introducing the Hamiltonian function $\mathcal{H}$,

$$
\mathcal{H}(t, \mathbf{x}, \mathbf{u}, \mathbf{p}, \boldsymbol{\lambda}, \boldsymbol{\mu}, \boldsymbol{\theta}):=\ell(t, \mathbf{x}, \mathbf{u}, \mathbf{p}, \boldsymbol{\theta})+\boldsymbol{\lambda}^{T} \mathbf{f}(t, \mathbf{x}, \mathbf{u}, \mathbf{p}, \boldsymbol{\theta})+\boldsymbol{\mu}^{T} \boldsymbol{\Omega}(t, \mathbf{x}, \mathbf{u}, \mathbf{p}, \boldsymbol{\theta}),
$$

and assuming that the problem $\mathrm{OC}\left(\boldsymbol{\theta}^{0}\right)$ is not abnormal, the following first-order NCOs must hold almost everywhere in $\left[t_{0}, t_{\mathrm{f}}\right][20,34]:^{1}$

$$
\begin{aligned}
\mathbf{0}= & \mathcal{H}_{\mathbf{u}}\left(t, \mathbf{x}^{*}(t), \mathbf{u}^{*}(t), \mathbf{p}, \boldsymbol{\lambda}^{*}(t), \boldsymbol{\theta}^{0}\right), \\
\mathbf{0}= & \int_{t_{0}}^{t_{\mathrm{f}}} \mathcal{H}_{\mathbf{p}}\left(t, \mathbf{x}^{*}(t), \mathbf{u}^{*}(t), \mathbf{p}, \boldsymbol{\lambda}^{*}(t), \boldsymbol{\theta}^{0}\right) d t+\varphi_{\mathbf{p}}\left(\mathbf{x}\left(t_{\mathrm{f}}\right), \mathbf{p}, \boldsymbol{\theta}\right) \\
& +\mathbf{T}_{\mathbf{p}}\left(\mathbf{x}^{*}\left(t_{\mathrm{f}}\right), \mathbf{p}, \boldsymbol{\theta}^{0}\right)^{T} \boldsymbol{\rho}^{*}+\mathbf{h}_{\mathbf{p}}(\mathbf{p}, \boldsymbol{\theta})^{T} \boldsymbol{\lambda}^{*}\left(t_{0}\right), \\
\dot{\boldsymbol{\lambda}}^{*}(t)= & -\mathcal{H}_{\mathbf{x}}\left(t, \mathbf{x}^{*}(t), \mathbf{u}^{*}(t), \mathbf{p}, \boldsymbol{\lambda}^{*}(t), \boldsymbol{\theta}^{0}\right), \\
\boldsymbol{\lambda}^{*}\left(t_{\mathrm{f}}\right)= & \varphi_{\mathbf{x}}\left(\mathbf{x}^{*}\left(t_{\mathrm{f}}\right), \mathbf{p}, \boldsymbol{\theta}^{0}\right)+\mathbf{T}_{\mathbf{x}}\left(\mathbf{x}^{*}\left(t_{\mathrm{f}}\right), \mathbf{p}, \boldsymbol{\theta}^{0}\right)^{T} \boldsymbol{\rho}^{*}, \\
0= & \rho_{i}^{*} T_{i}\left(\mathbf{x}^{*}\left(t_{\mathrm{f}}\right), \mathbf{p}, \boldsymbol{\theta}^{0}\right) \quad \forall i=1, \ldots, n_{\mathbf{T}}, \\
0 \leq & \rho_{i}^{*} \quad \forall i=1, \ldots, n_{\mathbf{T}}, \\
0= & \mu_{i}^{*}(t) \Omega_{i}\left(t, \mathbf{x}^{*}(t), \mathbf{u}^{*}(t), \mathbf{p}, \boldsymbol{\theta}^{0}\right) \quad \forall i=1, \ldots, n_{\boldsymbol{\Omega}}, \\
0 \leq & \mu_{i}^{*}(t) \quad \forall i=1, \ldots, n_{\boldsymbol{\Omega}}
\end{aligned}
$$

for some $\boldsymbol{\lambda}^{*}(t) \in \mathbb{R}^{n_{\mathbf{x}}}, \boldsymbol{\mu}^{*}(t) \in \mathbb{R}^{n_{\boldsymbol{\Omega}}}, t \in\left[t_{0}, t_{\mathrm{f}}\right]$, and $\boldsymbol{\rho}^{*} \in \mathbb{R}^{n_{\mathbf{T}}}$.

Two additional assumptions are made in the analysis that follows:

\footnotetext{
${ }^{1}$ The notation $\mathbf{y}_{\mathbf{z}}$ is used for the Jacobian matrix of the vector $\mathbf{y}$ with respect to the vector $\mathbf{z}$ $[25]$.
} 
1. The Hamiltonian function is regular, which implies that the optimal inputs $\mathbf{u}^{*}(t)$ are continuous on $\left[t_{0}, t_{\mathrm{f}}\right][28]$.

2. Strict complementarity slackness holds; that is,

(a) the multipliers $\rho_{i}^{*}$ corresponding to the active terminal constraints $T_{i}^{*}$ are strictly nonzero; and

(b) if $\left[a_{i k}, b_{i k}\right] \subset\left[t_{0}, t_{\mathrm{f}}\right]$ is an interval of maximal length on which the path constraint $\Omega_{i}^{*}$ is active, then the multiplier function $\mu_{i}^{*}(t)$ is strictly nonzero for each $t \in\left(a_{i k}, b_{i k}\right)$ [28].

Subsequently, the active path and terminal constraints are denoted by $\mathbf{T}^{a}$ and $\boldsymbol{\Omega}^{a}$, respectively, and their numbers by $n_{\mathbf{T}}^{a}$ and $n_{\boldsymbol{\Omega}}^{a}(t)$. The corresponding vectors of multipliers and multiplier functions are denoted by $\boldsymbol{\rho}^{a}$ and $\boldsymbol{\mu}^{a}$, respectively.

2.2. Solution structure and switching times. For problems having mixed control-state constraints, a constraint can be active over a number of different time intervals, meaning that the number of active path constraints may fluctuate over time. To describe this situation, we assume that the number of such time intervals remains finite in the optimal nominal solution. It follows that, for each $i=1, \ldots, n_{\Omega}$, there is a number $N_{i} \geq 0$ of disjoint intervals $\left[a_{i k}, b_{i k}\right] \subset\left[t_{0}, t_{\mathrm{f}}\right], k=1, \ldots, N_{i}$, such that

$$
\Omega_{i}\left(t, \mathbf{x}^{*}(t), \mathbf{u}^{*}(t), \mathbf{p}^{*}, \boldsymbol{\theta}^{0}\right)=0 \quad \forall t \in\left\{\left[a_{i 1}, b_{i 1}\right], \ldots,\left[a_{i N_{i}}, b_{i N_{i}}\right]\right\} .
$$

The time instants $a_{i k}$ and $b_{i k}$ are called the switching times of the constraint $\Omega_{i}$. The set of all switching times for the optimal nominal solution, together with the initial time $t_{0}$ and final time $t_{\mathrm{f}}$, are denoted collectively by

$$
\mathcal{T}:=\bigcup_{i \in\left\{1, \ldots, n_{\Omega}\right\}}\left\{a_{i 1}, b_{i 1}, \ldots, a_{i N_{i}}, b_{i N_{i}}\right\} \cup\left\{t_{0}, t_{\mathrm{f}}\right\} .
$$

Henceforth, the set $\mathcal{T}$ will simply be represented by $\mathcal{T}=\left\{t_{0}^{*}, \ldots, t_{N}^{*}\right\}$, with $t_{0}=t_{0}^{*}<$ $\cdots<t_{N}^{*}=t_{\mathrm{f}}$.

It is important to note that the set of active constraints in any subinterval $\left[t_{k}^{*}, t_{k+1}^{*}\right]$ remains constant, while the sets of active constraints in different subintervals $\left[t_{k}^{*}, t_{k+1}^{*}\right]$ and $\left[t_{l}^{*}, t_{l+1}^{*}\right]$ of $\left[t_{0}, t_{\mathrm{f}}\right]$ are generally different. That is, $\boldsymbol{\Omega}^{a}\left(t, \mathbf{x}^{*}(t), \mathbf{u}^{*}(t), \mathbf{p}^{*}, \boldsymbol{\theta}^{0}\right)$ will typically be a different vector function on different subintervals $\left[t_{k}^{*}, t_{k+1}^{*}\right]$ and $\left[t_{l}^{*}, t_{l+1}^{*}\right]$. To keep the notation simple, we still choose to keep the generic notation $\boldsymbol{\Omega}^{a}$ for the vector of active constraints on any subinterval of $\left[t_{0}, t_{\mathrm{f}}\right]$. In what follows, care will be been taken to associate each vector function $\boldsymbol{\Omega}^{a}$ with its corresponding subinterval.

3. Sensitivity- and constraint-seeking directions. The purpose of this section is to introduce the concepts of sensitivity- and constraint-seeking directions in the input space. To characterize these directions, small variations of the optimal inputs around their nominal optimal values $\left(\mathbf{u}^{*}, \mathbf{p}^{*}\right)$ are considered. Having characterized the class of input variations and the adaptation directions, the concept of selective input adaptation along each set of directions is then defined.

3.1. Class of input variations. Variations around the optimal nominal inputs $\left(\mathbf{u}^{*}, \mathbf{p}^{*}\right)$ are considered in the form

$$
\begin{aligned}
\tilde{\mathbf{u}}(t ; \eta) & =\mathbf{u}^{*}(t)+\eta \boldsymbol{\xi}^{\mathbf{u}}(t) \quad \forall t \in\left[t_{0}, t_{\mathrm{f}}\right] \\
\tilde{\mathbf{p}}(\eta) & =\mathbf{p}^{*}+\eta \boldsymbol{\xi}^{\mathbf{p}}
\end{aligned}
$$

where $\left(\boldsymbol{\xi}^{\mathbf{u}}, \boldsymbol{\xi}^{\mathbf{p}}\right) \in \hat{\mathcal{C}}\left[t_{0}, t_{\mathrm{f}}\right]^{n_{\mathbf{u}}} \times \mathbb{R}^{n_{\mathbf{p}}}$ are the input variation directions, with $\hat{\mathcal{C}}\left[t_{0}, t_{\mathrm{f}}\right]^{n_{\mathbf{u}}}$ the linear space of piecewise-continuous vector functions of dimension $n_{\mathbf{u}}$ on $\left[t_{0}, t_{\mathrm{f}}\right]$, and $\eta$ 

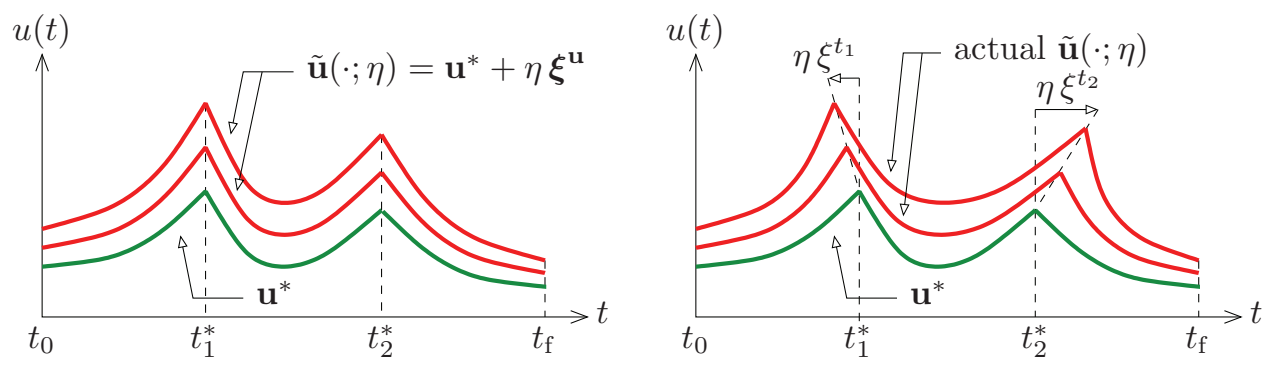

FIG. 1. Classes of input variations for the time-varying controls. Left plot: Variations in the control magnitude with fixed switching times. Right plot: Variations in both the control magnitude and switching times.

the magnitude of input variation, such that $|\eta| \ll 1$. The linear space $\hat{\mathcal{C}}\left[t_{0}, t_{\mathrm{f}}\right]^{n_{\mathbf{u}}} \times \mathbb{R}^{n_{\mathbf{p}}}$ subsequently will be referred to as the space of input variations.

Notice that the variation functions $\boldsymbol{\xi}^{\mathbf{u}}(t)$ can have a finite number of discontinuities on $\left[t_{0}, t_{\mathrm{f}}\right]$, for instance at the switching times $t_{k}^{*}, k=1, \ldots, N-1$, but the times at which a discontinuity occurs, together with the final time $t_{\mathrm{f}}=t_{N}^{*}$, are not varied with $\eta$. As illustrated in the left plot of Figure 1, such input variation functions act only on the magnitude of the nominal optimal inputs $\mathbf{u}^{*}(t)$, yet not on the associated switching times.

In practice, however, parametric perturbations typically cause variations in both the magnitude and the switching times of the optimal inputs as [30]

$$
\tilde{t}_{k}(\eta)=t_{k}^{*}+\eta \xi^{t_{k}}, \quad k=1, \ldots, N
$$

with $\xi^{t_{k}} \in \mathbb{R}$. This situation is depicted in the right plot on Figure 1. But this is not an actual limitation, for switching time variations can always be recast as parameter variations in an equivalent multistage dynamic system defined on constant time stages. One such reformulation is outlined subsequently.

Suppose that the perturbed inputs $\tilde{\mathbf{u}}(\cdot ; \eta)$ have varying switching times $\tilde{t}_{k}(\eta)$ given by (3.3). For a given $\eta \ll 1$ and any two consecutive switching times $\tilde{t}_{k-1}(\eta)<\tilde{t}_{k}(\eta)$, let the scaled time $t_{\mathrm{s}}$ be defined as

$$
t_{\mathrm{s}}=t_{k-1}^{*}+\left(t_{k}^{*}-t_{k-1}^{*}\right) \frac{t-\tilde{t}_{k-1}(\eta)}{\tilde{t}_{k}(\eta)-\tilde{t}_{k-1}(\eta)} .
$$

Define also the scaled control variables $\tilde{\mathbf{u}}_{\mathbf{s}}(\cdot ; \eta) \in \hat{\mathcal{C}}\left[t_{0}, t_{N}^{*}\right]^{n_{\mathbf{u}}}$ such that

$\tilde{\mathbf{u}}_{\mathbf{s}}\left(t_{\mathrm{s}} ; \eta\right)=\tilde{\mathbf{u}}\left(\tilde{t}_{k-1}(\eta)+\left[\tilde{t}_{k}(\eta)-\tilde{t}_{k-1}(\eta)\right] \frac{t_{\mathrm{s}}-t_{k-1}^{*}}{t_{k}^{*}-t_{k-1}^{*}} ; \eta\right) \forall t_{\mathrm{s}} \in\left(t_{k-1}^{*}, t_{k}^{*}\right], k=1, \ldots, N$.

Clearly, $\tilde{\mathbf{u}}\left(\tilde{t}_{k}(\eta) ; \eta\right)=\tilde{\mathbf{u}}_{\mathrm{s}}\left(t_{k}^{*} ; \eta\right)$ for all $|\eta| \ll 1$, and so the switching times of $\tilde{\mathbf{u}}_{\mathrm{s}}$ are constant.

Now, consider variations of the scaled controls $\mathbf{u}_{\mathrm{s}}$ in the directions $\boldsymbol{\xi}^{\mathbf{u}_{\mathrm{s}}} \in \hat{\mathcal{C}}\left[0, t_{N}^{*}\right]^{n_{\mathbf{u}}}$ as

$$
\tilde{\mathbf{u}}_{\mathrm{s}}\left(t_{\mathrm{s}} ; \eta\right)=\mathbf{u}_{\mathrm{s}}^{*}\left(t_{\mathrm{s}}\right)+\eta \boldsymbol{\xi}^{\mathbf{u}_{\mathrm{s}}}\left(t_{\mathrm{s}}\right) \forall t_{\mathrm{s}} \in\left(t_{k-1}^{*}, t_{k}^{*}\right], k=1, \ldots, N,
$$

together with switching time variations as in (3.3). The combined effect of input 
variations along $\boldsymbol{\xi}^{\mathbf{u}_{\mathrm{s}}}$ and $\xi^{t_{k}}$ on $\tilde{\mathbf{u}}(\cdot ; \eta)$ is

$$
\begin{aligned}
\tilde{\mathbf{u}}(t ; \eta)= & \mathbf{u}^{*}\left(t_{k-1}^{*}+\left(t_{k}^{*}-t_{k-1}^{*}\right) \frac{t-t_{k-1}^{*}-\eta \xi^{t_{k-1}}}{t_{k}^{*}-t_{k-1}^{*}+\eta\left(\xi^{t_{k}}-\xi^{t_{k-1}}\right)}\right) \\
& +\eta \boldsymbol{\xi}^{\mathbf{u}_{\mathrm{s}}}\left(t_{k-1}^{*}+\left(t_{k}^{*}-t_{k-1}^{*}\right) \frac{t-t_{k-1}^{*}-\eta \xi^{t_{k-1}}}{t_{k}^{*}-t_{k-1}^{*}+\eta\left(\xi^{t_{k}}-\xi^{t_{k-1}}\right)}\right)
\end{aligned}
$$

for all $t \in\left(t_{k-1}^{*}+\eta \xi^{t_{k-1}}, t_{k}^{*}+\eta \xi^{t_{k}}\right], k=1, \ldots, N$. In particular, the perturbed inputs $\tilde{\mathbf{u}}(t ; \eta)$ in $(3.5)$ are now able to represent variations as shown in the right plot of Figure 1 , and they naturally reduce to (3.1) when selecting $\xi^{t_{k}}=0$ for all $k=1, \ldots, N$.

Similarly, scaled state variables can be introduced as

$\tilde{\mathbf{x}}_{\mathbf{s}}\left(t_{\mathrm{s}} ; \eta\right)=\tilde{\mathbf{x}}\left(\tilde{t}_{k-1}(\eta)+\left[\tilde{t}_{k}(\eta)-\tilde{t}_{k-1}(\eta)\right] \frac{t_{\mathrm{s}}-t_{k-1}^{*}}{t_{k}^{*}-t_{k-1}^{*}} ; \eta\right) \forall t_{\mathrm{s}} \in\left(t_{k-1}^{*}, t_{k}^{*}\right], k=1, \ldots, N$.

These variables satisfy the following auxiliary system of ODEs:

$$
\dot{\tilde{\mathbf{x}}}_{\mathrm{s}}\left(t_{\mathrm{s}} ; \eta\right)=\mathbf{f}_{\mathrm{s}}\left(t_{\mathrm{s}}, \tilde{\mathbf{x}}_{\mathrm{s}}\left(t_{\mathrm{s}} ; \eta\right), \tilde{\mathbf{u}}_{\mathrm{s}}\left(t_{\mathrm{s}} ; \eta\right), \tilde{\mathbf{p}}_{\mathrm{s}}(\eta), \boldsymbol{\theta}\right),
$$

on each time stage $\left(t_{k-1}^{*}, t_{k}^{*}\right], k=1, \ldots, N$, where $\tilde{\mathbf{p}}_{\mathbf{s}}(\eta)^{T}=\left[\tilde{\mathbf{p}}(\eta)^{T} \tilde{t}_{1}(\eta) \cdots \tilde{t}_{N}(\eta)\right] \in$ $\mathbb{R}^{n_{p}+N}$ is the extended time-invariant input vector, and the vector function $\mathbf{f}_{\mathrm{s}}$ is defined as

$$
\mathbf{f}_{\mathrm{s}}\left(t_{\mathrm{s}}, \mathbf{x}_{\mathrm{s}}, \mathbf{u}_{\mathrm{s}}, \mathbf{p}_{\mathrm{s}}, \boldsymbol{\theta}\right):=\left[\tilde{t}_{k}-\tilde{t}_{k-1}\right] \mathbf{f}\left(\tilde{t}_{k-1}+\left[\tilde{t}_{k}-\tilde{t}_{k-1}\right] \frac{t_{\mathrm{s}}-t_{k-1}^{*}}{t_{k}^{*}-t_{k-1}^{*}}, \mathbf{x}_{\mathrm{s}}, \mathbf{u}_{\mathrm{s}}, \mathbf{p}, \boldsymbol{\theta}\right) .
$$

After applying the aforementioned rescaling, an equivalent optimal control problem is obtained in the input variables $\left(\mathbf{u}_{\mathrm{s}}, \mathbf{p}_{\mathrm{s}}\right)$ and the state variables $\mathbf{x}_{\mathrm{s}}$. By construction, a nominal optimal solution of this auxiliary problem is such that $\mathbf{u}_{\mathbf{s}}^{*}(t)=\mathbf{u}^{*}(t)$ for all $t \in\left(t_{k-1}^{*}, t_{k}^{*}\right], k=1, \ldots, N$, and $\mathbf{p}_{\mathrm{s}}^{* T}=\left[\mathbf{p}^{* T} t_{1}^{*} \cdots t_{N}^{*}\right]$.

Since the switching times of $\tilde{\mathbf{u}}_{\mathbf{s}}(\cdot ; \eta)$ remain constant in $\left[t_{0}, t_{N}^{*}\right]$, equal to $t_{k}^{*}$, regardless of the variation magnitude $\eta$, variations of the form of (3.1) that do not act on the switching times can safely be applied to these scaled inputs. An application of this rescaling procedure is given later in Example 3.2.

Without loss of generality, we shall concentrate on the class of input variations (3.1), (3.2) in the rest of the paper.

3.2. Directions of invariance. Consider the perturbed inputs (3.1), (3.2), and let the corresponding perturbed states be denoted by $\tilde{\mathbf{x}}(t ; \eta)$, so that the triplet $(\tilde{\mathbf{x}}(\cdot ; \eta), \tilde{\mathbf{u}}(\cdot ; \eta), \tilde{\mathbf{p}}(\eta))$ satisfies $(2.2)$ for the parameter values $\boldsymbol{\theta}^{0}$. By continuous differentiability of $\mathbf{f}$ with respect to the inputs and states at $\left(\mathbf{x}^{*}(t), \mathbf{u}^{*}(t), \mathbf{p}^{*}\right)$, a first-order Taylor expansion of $\mathbf{f}$ around this point gives $^{2}$

$$
\begin{aligned}
\dot{\tilde{\mathbf{x}}}(t ; \eta)-\dot{\mathbf{x}}^{*}(t) & =\mathbf{f}\left(t, \tilde{\mathbf{x}}(t ; \eta), \tilde{\mathbf{u}}(t ; \eta), \tilde{\mathbf{p}}(\eta), \boldsymbol{\theta}^{0}\right)-\mathbf{f}\left(t, \mathbf{x}^{*}(t), \mathbf{u}^{*}(t), \mathbf{p}^{*}, \boldsymbol{\theta}^{0}\right) \\
& =\mathbf{f}_{\mathbf{x}}^{*}[t]\left(\tilde{\mathbf{x}}(t ; \eta)-\mathbf{x}^{*}(t)\right)+\eta\left(\mathbf{f}_{\mathbf{u}}^{*}[t] \boldsymbol{\xi}^{\mathbf{u}}(t)+\mathbf{f}_{\mathbf{p}}^{*}[t] \boldsymbol{\xi}^{\mathbf{p}}\right)+O\left(\eta^{2}\right) .
\end{aligned}
$$

A first-order approximation of $\tilde{\mathbf{x}}(t ; \eta)$ is thus obtained as

$$
\tilde{\mathbf{x}}(t ; \eta)=\mathbf{x}^{*}(t)+\eta \boldsymbol{\xi}^{\mathbf{x}}(t)+O\left(\eta^{2}\right)
$$

\footnotetext{
${ }^{2}$ The following compact notation is used throughout the paper: $y^{*}[t]:=y\left(t, \mathbf{x}^{*}(t), \mathbf{u}^{*}(t), \mathbf{p}^{*}, \boldsymbol{\theta}^{0}\right)$ and $z^{*}\left[t_{\mathrm{f}}\right]:=z\left(\mathbf{x}^{*}\left(t_{\mathrm{f}}\right), \mathbf{p}^{*}, \boldsymbol{\theta}^{0}\right)$.
} 
where $\boldsymbol{\xi}^{\mathbf{x}}(t)$ satisfies

$$
\begin{aligned}
\dot{\boldsymbol{\xi}}^{\mathbf{x}}(t) & =\mathbf{f}_{\mathbf{x}}^{*}[t] \boldsymbol{\xi}^{\mathbf{x}}(t)+\mathbf{f}_{\mathbf{u}}^{*}[t] \boldsymbol{\xi}^{\mathbf{u}}(t)+\mathbf{f}_{\mathbf{p}}^{*}[t] \boldsymbol{\xi}^{\mathbf{p}} & \forall t \in\left[t_{k-1}^{*}, t_{k}^{*}\right), k=1, \ldots, N \\
\boldsymbol{\xi}^{\mathbf{x}}\left(t_{0}\right) & =\mathbf{h}_{\mathbf{p}}^{*} \boldsymbol{\xi}^{\mathbf{p}}, \quad \boldsymbol{\xi}^{\mathbf{x}}\left(t_{k}^{*+}\right)=\boldsymbol{\xi}^{\mathbf{x}}\left(t_{k}^{*-}\right), & k=1, \ldots, N-1 .
\end{aligned}
$$

The unique solution to the above linear system can be written in the form [32]

$$
\begin{aligned}
\boldsymbol{\xi}^{\mathbf{x}}(t)= & \boldsymbol{\Phi}^{\mathbf{f}_{\mathbf{x}}^{*}}\left(t, t_{k-1}^{*}\right) \boldsymbol{\xi}^{\mathbf{x}}\left(t_{k-1}^{*}\right)+\int_{t_{k-1}^{*}}^{t} \boldsymbol{\Phi}_{\mathbf{x}}^{\mathbf{f}_{\mathbf{x}}^{*}}(t, s)\left(\mathbf{f}_{\mathbf{u}}^{*}[s] \boldsymbol{\xi}^{\mathbf{u}}(s)+\mathbf{f}_{\mathbf{p}}^{*}[s] \boldsymbol{\xi}^{\mathbf{p}}\right) d s \\
= & \boldsymbol{\Phi}^{\mathbf{f}_{\mathbf{x}}^{*}}\left(t, t_{0}\right) \mathbf{h}_{\mathbf{p}}^{*} \boldsymbol{\xi}^{\mathbf{p}}+\sum_{i=1}^{k-1} \int_{t_{i-1}^{*}}^{t_{i}^{*}} \boldsymbol{\Phi}^{\mathbf{f}_{\mathbf{x}}^{*}}(t, s)\left(\mathbf{f}_{\mathbf{u}}^{*}[s] \boldsymbol{\xi}^{\mathbf{u}}(s)+\mathbf{f}_{\mathbf{p}}^{*}[s] \boldsymbol{\xi}^{\mathbf{p}}\right) d s \\
& +\int_{t_{k-1}^{*}}^{t} \boldsymbol{\Phi}^{\mathbf{f}_{\mathbf{x}}^{*}}(t, s)\left(\mathbf{f}_{\mathbf{u}}^{*}[s] \boldsymbol{\xi}^{\mathbf{u}}(s)+\mathbf{f}_{\mathbf{p}}^{*}[s] \boldsymbol{\xi}^{\mathbf{p}}\right) d s
\end{aligned}
$$

for each $t \in\left(t_{k-1}^{*}, t_{k}^{*}\right], k=1, \ldots, N$, where $\mathbf{\Phi}^{\mathbf{A}}(t, s)$ stands for the state-transition matrix of the homogeneous linear system

$$
\dot{\mathbf{z}}(t)=\mathbf{A}(t) \mathbf{z}(t) \forall t \geq t_{0}, \quad \mathbf{z}\left(t_{0}\right)=\mathbf{z}_{0} .
$$

Next, consider a general functional $\boldsymbol{\psi}: \hat{\mathcal{C}}\left[t_{0}, t_{\mathrm{f}}\right]^{n_{\mathbf{u}}} \times \mathbb{R}^{n_{\mathbf{p}}} \rightarrow \mathbb{R}^{n_{\psi}}$ defined as

$$
\boldsymbol{\psi}(\mathbf{u}, \mathbf{p}):=\mathbf{\Psi}(\underline{t}, \mathbf{x}(\underline{t}), \mathbf{u}(\underline{t}), \mathbf{p}, \boldsymbol{\theta}),
$$

with $\underline{t}$ a fixed time in $\left(t_{k-1}^{*}, t_{k}^{*}\right]$, for some $k \in\{1, \ldots, N\}$. The variation in the functional $\boldsymbol{\psi}$ caused by the input variation (3.1), (3.2) can be obtained as the Gâteaux derivative $[8,35]$ of $\boldsymbol{\psi}$ in the direction $\left(\boldsymbol{\xi}^{\mathbf{u}}, \boldsymbol{\xi}^{\mathbf{p}}\right)$ at $\left(\mathbf{u}^{*}, \mathbf{p}^{*}\right)$,

$$
\begin{aligned}
\delta \boldsymbol{\psi}\left(\mathbf{u}^{*}, \mathbf{p}^{*} ; \boldsymbol{\xi}^{\mathbf{u}}, \boldsymbol{\xi}^{\mathbf{p}}\right): & =\left.\frac{\partial}{\partial \eta} \boldsymbol{\Psi}\left(\underline{t}, \tilde{\mathbf{x}}(\underline{t} ; \eta), \tilde{\mathbf{u}}(\underline{t} ; \eta), \tilde{\mathbf{p}}(\eta), \boldsymbol{\theta}^{0}\right)\right|_{\eta=0} \\
& =\boldsymbol{\Psi}_{\mathbf{x}}^{*}[\underline{t}] \boldsymbol{\xi}^{\mathbf{x}}(\underline{t})+\boldsymbol{\Psi}_{\mathbf{u}}^{*}[\underline{t}] \boldsymbol{\xi}^{\mathbf{u}}(\underline{t})+\boldsymbol{\Psi}_{\mathbf{p}}^{*}[\underline{t}] \boldsymbol{\xi}^{\mathbf{p}} .
\end{aligned}
$$

Using (3.9), this variation can be rewritten as

$$
\delta \boldsymbol{\psi}\left(\mathbf{u}^{*}, \mathbf{p}^{*} ; \boldsymbol{\xi}^{\mathbf{u}}, \boldsymbol{\xi}^{\mathbf{p}}\right)=\mathcal{D}_{\mathbf{\Psi}, \underline{t}}^{*}\left(\boldsymbol{\xi}^{\mathbf{u}}, \boldsymbol{\xi}^{\mathbf{p}}\right),
$$

where the linear operator $\mathcal{D}_{\mathbf{\Psi}, \underline{t}}^{*}: \hat{\mathcal{C}}\left[t_{0}, t_{\mathrm{f}}\right]^{n_{\mathbf{u}}} \times \mathbb{R}^{n_{\mathbf{p}}} \rightarrow \mathbb{R}$ is given by

$$
\begin{aligned}
\mathcal{D}_{\mathbf{\Psi}, \underline{t}}^{*}\left(\boldsymbol{\xi}^{\mathbf{u}}, \boldsymbol{\xi}^{\mathbf{p}}\right):= & \boldsymbol{\Psi}_{\mathbf{x}}^{*}[\underline{t}]\left[\boldsymbol{\Phi}^{\mathbf{f}_{\times}^{*}}\left(\underline{t}, t_{0}\right) \mathbf{h}_{\mathbf{p}}^{*} \boldsymbol{\xi}^{\mathbf{p}}+\sum_{i=1}^{k-1} \int_{t_{i-1}^{*}}^{t_{i}^{*}} \boldsymbol{\Phi}^{\mathbf{f}_{\times}^{*}}(\underline{t}, s)\left[\mathbf{f}_{\mathbf{u}}^{*}[s] \boldsymbol{\xi}^{\mathbf{u}}(s)+\mathbf{f}_{\mathbf{p}}^{*}[s] \boldsymbol{\xi}^{\mathbf{p}}\right] d s\right. \\
& \left.+\int_{t_{k-1}^{*}}^{\underline{\underline{t}}} \boldsymbol{\Phi}^{\mathbf{f}_{\times}^{*}}(\underline{t}, s)\left[\mathbf{f}_{\mathbf{u}}^{*}[s] \boldsymbol{\xi}^{\mathbf{u}}(s)+\mathbf{f}_{\mathbf{p}}^{*}[s] \boldsymbol{\xi}^{\mathbf{p}}\right] d s\right]+\boldsymbol{\Psi}_{\mathbf{u}}^{*}[\underline{t}] \boldsymbol{\xi}^{\mathbf{u}}(\underline{t})+\mathbf{\Psi}_{\mathbf{p}}^{*}[\underline{t}] \boldsymbol{\xi}^{\mathbf{p}} .
\end{aligned}
$$

If the value of $\boldsymbol{\psi}$ remains unaffected by a small variation in the direction $\left(\boldsymbol{\xi}^{\mathbf{u}}, \boldsymbol{\xi}^{\mathbf{p}}\right)$ at $\left(\mathbf{u}^{*}, \mathbf{p}^{*}\right)$, then $\left(\boldsymbol{\xi}^{\mathbf{u}}, \boldsymbol{\xi}^{\mathbf{p}}\right)$ is called a direction of invariance of $\boldsymbol{\psi}$ at $\left(\mathbf{u}^{*}, \mathbf{p}^{*}\right)$. This concept is formalized in the following definition.

Definition 3.1 (direction of invariance). The pair $\left(\boldsymbol{\xi}^{\mathbf{u}}, \boldsymbol{\xi}^{\mathbf{p}}\right) \in \hat{\mathcal{C}}\left[t_{0}, t_{\mathrm{f}}\right]^{n_{\mathbf{u}}} \times \mathbb{R}^{n_{\mathbf{p}}}$ is called a direction of invariance for the functional $\boldsymbol{\psi}$ at $\left(\mathbf{u}^{*}, \mathbf{p}^{*}\right)$ and for $\underline{t} \in\left[t_{0}, t_{\mathrm{f}}\right]$ if $\mathcal{D}_{\mathbf{\Psi}, \underline{t}}^{*}\left(\xi^{\mathbf{u}}, \xi^{\mathbf{p}}\right)=\mathbf{0}$. 
Clearly, any linear combination of directions of invariance for the functional $\psi$ is itself a direction of invariance for $\boldsymbol{\psi}$. Therefore, the set of all directions of invariance for $\boldsymbol{\psi}$, denoted by

$$
\mathcal{V}_{\boldsymbol{\Psi}, \underline{t}}^{*}:=\left\{\left(\boldsymbol{\xi}^{\mathbf{u}}, \boldsymbol{\xi}^{\mathbf{p}}\right) \in \hat{\mathcal{C}}\left[t_{0}, t_{\mathrm{f}}\right]^{n_{\mathbf{u}}} \times \mathbb{R}^{n_{\mathbf{p}}}: \mathcal{D}_{\boldsymbol{\Psi}, \underline{t}}^{*}\left(\boldsymbol{\xi}^{\mathbf{u}}, \boldsymbol{\xi}^{\mathbf{p}}\right)=\mathbf{0}\right\},
$$

is a linear subspace of $\hat{\mathcal{C}}\left[t_{0}, t_{\mathrm{f}}\right]^{n_{\mathbf{u}}} \times \mathbb{R}^{n_{\mathbf{p}}}$.

The following examples illustrate that the kernel of $\mathcal{D}_{\boldsymbol{\Psi}, t}^{*}\left(\boldsymbol{\xi}^{\mathbf{u}}, \boldsymbol{\xi}^{\mathbf{p}}\right)$ is nontrivial; i.e., the kernel contains elements other than the zero element. Moreover, the first example establishes that such kernels can be infinite dimensional, whereas the second example considers the problem of a varying end-time and applies the rescaling procedure in subsection 3.1.

Example 3.1. Consider the scalar dynamic system $\dot{x}(t)=-x(t)+u(t)$ for $t \in$ $[0,1]$, with parametric initial condition $x(0)=p$. We are interested in directions of invariance $\left(\xi^{u}, \xi^{p}\right)$ of the functional $\boldsymbol{\psi}: \hat{\mathcal{C}}[0,1] \times \mathbb{R} \rightarrow \mathbb{R}$ defined by $\boldsymbol{\psi}(u, p)=x(1)$. The linear operator $\mathcal{D}_{x, 1}^{*}$ is given by

$$
\mathcal{D}_{x, 1}^{*}\left(\xi^{u}, \xi^{p}\right)=\mathrm{e}^{-1}\left[\xi^{p}+\int_{0}^{1} \mathrm{e}^{s} \xi^{u}(s) d s\right] .
$$

In particular, for the constant variation function $\xi^{u}(t)=\alpha, 0 \leq t \leq 1$, we have $\mathcal{D}_{x, 1}^{*}\left(\xi^{u}, \xi^{p}\right)=\mathrm{e}^{-1} \xi^{p}+\left[1-\mathrm{e}^{-1}\right] \alpha$. It follows that $(t \mapsto \alpha,(1-\mathrm{e}) \alpha)$ is a direction of invariance of $\boldsymbol{\psi}$. Likewise, it is not hard to show that $(t \mapsto \alpha t,-\alpha)$ and $\left(t \mapsto \alpha t^{2}\right.$, $(2-\mathrm{e}) \alpha)$ are directions of invariance. More generally, by a simple induction on $n \geq 1$, one proves that if $\left(t \mapsto \alpha t^{n-1}, \beta\right)$ is a direction of invariance, then $\left(t \mapsto \alpha t^{n},-\mathrm{e} \alpha-n \beta\right)$ is also a direction of invariance. It follows that $\mathcal{V}_{x, 1}^{*}$ is an infinite-dimensional linear subspace of $\hat{\mathcal{C}}[0,1] \times \mathbb{R}$.

Example 3.2. Consider the functional $\boldsymbol{\psi}: \hat{\mathcal{C}}\left[0, t_{\mathrm{f}}\right] \times \mathbb{R} \rightarrow \mathbb{R}$ defined by $\boldsymbol{\psi}\left(u, t_{\mathrm{f}}\right)=$ $x\left(t_{\mathrm{f}}\right)$, where $x(t)$ is a solution of the scalar dynamic system $\dot{x}(t)=-x(t)+u(t)$ for $t \in\left[0, t_{\mathrm{f}}\right]$, with initial condition $x(0)=1$. In order to analyze the directions of invariance of $\boldsymbol{\psi}$, we first consider the scaled time $t_{s}:=\frac{t}{t_{\mathrm{f}}}$ and apply a rescaling as described in subsection 3.1,

$$
\dot{x}_{\mathrm{s}}\left(t_{\mathrm{s}}\right)=t_{\mathrm{f}}\left(-x_{\mathrm{s}}\left(t_{\mathrm{s}}\right)+u_{\mathrm{s}}\left(t_{\mathrm{s}}\right)\right) \quad \forall t_{\mathrm{s}} \in[0,1] .
$$

The linear operator $\mathcal{D}_{x_{s}, 1}^{*}$ is given by

$$
\mathcal{D}_{x_{\mathrm{s}}, 1}^{*}\left(\xi^{u_{\mathrm{s}}}, \xi^{t_{\mathrm{f}}}\right)=\int_{0}^{t_{\mathrm{f}}^{*}} \mathrm{e}^{s-t_{\mathrm{f}}^{*}}\left[t_{\mathrm{f}}^{*} \xi^{u_{\mathrm{s}}}(s)+u_{\mathrm{s}}^{*}(s) \xi^{t_{\mathrm{f}}}\right] d s .
$$

It follows that, for any real scalar $\alpha,\left(t_{\mathrm{s}} \mapsto-\frac{u_{\mathrm{s}}^{*}\left(t_{\mathrm{s}}\right)}{t_{\mathrm{f}}} \alpha, \alpha\right)$ is a direction of invariance of $\psi$ at $\left(u_{\mathrm{s}}^{*}, t_{\mathrm{f}}^{*}\right)$ for the scaled problem.

3.3. Characterizing sensitivity- and constraint-seeking directions. A sensitivity-seeking (SS) direction at the nominal optimal solution $\left(\mathbf{u}^{*}, \mathbf{p}^{*}\right)$ corresponds to a direction $\left(\boldsymbol{\xi}^{\mathbf{u}}, \boldsymbol{\xi}^{\mathbf{p}}\right)$ in the space of input variations along which an infinitesimal variation from $\left(\mathbf{u}^{*}, \mathbf{p}^{*}\right)$ leaves all of the active constraints unchanged. A formal definition can now be provided based on the concept of direction of invariance introduced previously.

Definition 3.2 (sensitivity-seeking directions). The pair $\left(\boldsymbol{\xi}^{\mathbf{u}}, \boldsymbol{\xi}^{\mathbf{p}}\right) \in \hat{\mathcal{C}}\left[t_{0}, t_{\mathrm{f}}\right]^{n_{\mathbf{u}}} \times$ $\mathbb{R}^{n_{\mathbf{p}}}$ is called an SS direction for the optimal control problem $O C\left(\boldsymbol{\theta}^{0}\right)$ at $\left(\mathbf{u}^{*}, \mathbf{p}^{*}\right)$ if $\left(\boldsymbol{\xi}^{\mathbf{u}}, \boldsymbol{\xi}^{\mathbf{p}}\right)$ is a direction of invariance for 
1. the active terminal constraints $\mathbf{T}^{a}\left(t_{\mathrm{f}}, \tilde{\mathbf{x}}\left(t_{\mathrm{f}} ; \eta\right), \tilde{\mathbf{p}}(\eta), \boldsymbol{\theta}^{0}\right)$,

$$
\begin{aligned}
\mathbf{0}= & \mathcal{D}_{\mathbf{T}^{a}, t_{\mathrm{f}}}^{*}\left(\boldsymbol{\xi}^{\mathbf{u}}, \boldsymbol{\xi}^{\mathbf{p}}\right) \\
= & \mathbf{T}_{\mathbf{x}}^{a}\left[t_{\mathrm{f}}\right]\left[\mathbf{\Phi}^{\mathbf{f}_{\times}^{*}}\left(t_{\mathrm{f}}, t_{0}\right) \mathbf{h}_{\mathbf{p}}^{*} \boldsymbol{\xi}^{\mathbf{p}}+\sum_{k=1}^{N} \int_{t_{k-1}^{*}}^{t_{k}^{*}} \boldsymbol{\Phi}^{\mathbf{f}_{\times}^{*}}\left(t_{\mathrm{f}}, s\right)\left[\mathbf{f}_{\mathbf{u}}^{*}[s] \boldsymbol{\xi}^{\mathbf{u}}(s)+\mathbf{f}_{\mathbf{p}}^{*}[s] \boldsymbol{\xi}^{\mathbf{p}}\right] d s\right] \\
& +\mathbf{T}_{\mathbf{p}}^{a}\left[t_{\mathrm{f}}\right] \boldsymbol{\xi}^{\mathbf{p}} ; \text { and }
\end{aligned}
$$

2. the active path constraints $\boldsymbol{\Omega}^{a}\left(t, \tilde{\mathbf{x}}(t ; \eta), \tilde{\mathbf{u}}(t ; \eta), \tilde{\mathbf{p}}(\eta), \boldsymbol{\theta}^{0}\right)$,

$$
\begin{aligned}
\mathbf{0}= & \mathcal{D}_{\boldsymbol{\Omega}^{a}, t}^{*}\left(\boldsymbol{\xi}^{\mathbf{u}}, \boldsymbol{\xi}^{\mathbf{p}}\right) \\
= & \boldsymbol{\Omega}_{\mathbf{x}}^{a}[t]\left[\boldsymbol{\Phi}^{\mathbf{f}_{\times}^{*}}\left(t, t_{0}\right) \mathbf{h}_{\mathbf{p}}^{*} \boldsymbol{\xi}^{\mathbf{p}}+\sum_{i=1}^{k-1} \int_{t_{i-1}^{*}}^{t_{i}^{*}} \boldsymbol{\Phi}^{\mathbf{f}_{\times}^{*}}(t, s)\left[\mathbf{f}_{\mathbf{u}}^{*}[s] \boldsymbol{\xi}^{\mathbf{u}}(s)+\mathbf{f}_{\mathbf{p}}^{*}[s] \boldsymbol{\xi}^{\mathbf{p}}\right] d s\right. \\
& \left.+\int_{t_{k-1}^{*}}^{t} \boldsymbol{\Phi}^{\mathbf{f}_{\times}^{*}}(t, s)\left[\mathbf{f}_{\mathbf{u}}^{*}[s] \boldsymbol{\xi}^{\mathbf{u}}(s)+\mathbf{f}_{\mathbf{p}}^{*}[s] \boldsymbol{\xi}^{\mathbf{p}}\right] d s\right]+\boldsymbol{\Omega}_{\mathbf{u}}^{a}[t] \boldsymbol{\xi}^{\mathbf{u}}(t)+\boldsymbol{\Omega}_{\mathbf{p}}^{a}[t] \boldsymbol{\xi}^{\mathbf{p}},
\end{aligned}
$$

at each $t \in\left(t_{k-1}^{*}, t_{k}^{*}\right], k=1, \ldots, N$.

Let the set of sensitivity-seeking (SS) directions for $\operatorname{OC}\left(\boldsymbol{\theta}^{0}\right)$ at $\left(\mathbf{u}^{*}, \mathbf{p}^{*}\right)$ be denoted by

$$
\begin{aligned}
\mathcal{V}_{s}^{*} & :=\left\{\left(\boldsymbol{\xi}^{\mathbf{u}}, \boldsymbol{\xi}^{\mathbf{p}}\right) \in \hat{\mathcal{C}}\left[t_{0}, t_{\mathrm{f}}\right]^{n_{\mathbf{u}}} \times \mathbb{R}^{n_{\mathbf{p}}}: \mathcal{D}_{\mathbf{T}^{a}, t_{\mathrm{f}}}^{*}\left(\boldsymbol{\xi}^{\mathbf{u}}, \boldsymbol{\xi}^{\mathbf{p}}\right)=\mathbf{0} ; \mathcal{D}_{\boldsymbol{\Omega}^{a}, t}^{*}\left(\boldsymbol{\xi}^{\mathbf{u}}, \boldsymbol{\xi}^{\mathbf{p}}\right)=\mathbf{0}, t \in\left[t_{0}, t_{\mathrm{f}}\right]\right\} \\
& =\mathcal{V}_{\mathbf{T}^{a}, t_{\mathrm{f}}}^{*} \cap\left(\bigcap_{t \in\left[t_{0}, t_{\mathrm{f}}\right]} \mathcal{V}_{\boldsymbol{\Omega}^{a}, t}^{*}\right) .
\end{aligned}
$$

Clearly, $\mathcal{V}_{s}^{*}$ is a linear subspace of $\hat{\mathcal{C}}\left[t_{0}, t_{\mathrm{f}}\right]^{n_{\mathbf{u}}} \times \mathbb{R}^{n_{\mathbf{p}}}$ by the properties of the sets of invariance $\mathcal{V}_{\mathbf{T}^{a}, t_{\mathrm{f}}}^{*}$ and $\mathcal{V}_{\Omega^{a}, t}^{*}$. It is referred to as the $S S$ subspace for $\operatorname{OC}\left(\boldsymbol{\theta}^{0}\right)$ at $\left(\mathbf{u}^{*}, \mathbf{p}^{*}\right)$ thereafter.

Next, constraint-seeking (CS) directions are defined as those directions in $\hat{\mathcal{C}}\left[t_{0}, t_{\mathrm{f}}\right]^{n_{\mathbf{u}}} \times \mathbb{R}^{n_{\mathbf{p}}}$ which are orthogonal to the SS subspace.

DEFINITION 3.3 (constraint-seeking directions). The pair $\left(\boldsymbol{\xi}^{\mathbf{u}}, \boldsymbol{\xi}^{\mathbf{p}}\right) \in \hat{\mathcal{C}}\left[t_{0}, t_{\mathrm{f}}\right]^{n_{\mathbf{u}}} \times$ $\mathbb{R}^{n_{\mathbf{p}}}$ is called a CS direction for the optimal control problem $O C\left(\boldsymbol{\theta}^{0}\right)$ at $\left(\mathbf{u}^{*}, \mathbf{p}^{*}\right)$ if $\left(\boldsymbol{\xi}^{\mathbf{u}}, \boldsymbol{\xi}^{\mathbf{p}}\right)$ is orthogonal to $\mathcal{V}_{s}^{*}$; that is,

$$
0=\left\langle\left(\boldsymbol{\xi}^{\mathbf{u}}, \boldsymbol{\xi}^{\mathbf{p}}\right),\left(\boldsymbol{\zeta}^{\mathbf{u}}, \boldsymbol{\zeta}^{\mathbf{p}}\right)\right\rangle \quad \forall\left(\boldsymbol{\zeta}^{\mathbf{u}}, \boldsymbol{\zeta}^{\mathbf{p}}\right) \in \mathcal{V}_{s}^{*}
$$

where $\langle\cdot, \cdot\rangle$ stands for any inner product on $\hat{\mathcal{C}}\left[t_{0}, t_{\mathrm{f}}\right]^{n_{\mathbf{u}}} \times \mathbb{R}^{n_{\mathbf{p}}}$.

Remark 3.1. The following inner product on $\hat{\mathcal{C}}\left[t_{0}, t_{\mathrm{f}}\right]^{n_{\mathbf{u}}} \times \mathbb{R}^{n_{\mathbf{p}}}$ is considered subsequently to define the CS directions:

$$
\left\langle\left(\boldsymbol{\xi}^{\mathbf{u}}, \boldsymbol{\xi}^{\mathbf{p}}\right),\left(\boldsymbol{\zeta}^{\mathbf{u}}, \zeta^{\mathbf{p}}\right)\right\rangle:=\int_{t_{0}}^{t_{\mathrm{f}}} \boldsymbol{\xi}^{\mathbf{u}}(t)^{T} \boldsymbol{\zeta}^{\mathbf{u}}(t) d t+\boldsymbol{\xi}^{\mathbf{p}^{T}} \boldsymbol{\zeta}^{\mathbf{p}}
$$

for $\left(\boldsymbol{\xi}^{\mathbf{u}}, \boldsymbol{\xi}^{\mathbf{p}}\right),\left(\boldsymbol{\zeta}^{\mathbf{u}}, \boldsymbol{\zeta}^{\mathbf{p}}\right) \in \hat{\mathcal{C}}\left[t_{0}, t_{\mathrm{f}}\right]^{n_{\mathbf{u}}} \times \mathbb{R}^{n_{\mathbf{p}}}$.

Denote the set of all CS directions for $\operatorname{OC}\left(\boldsymbol{\theta}^{0}\right)$ at $\left(\mathbf{u}^{*}, \mathbf{p}^{*}\right)$ by $\mathcal{V}_{c}^{*}$. By the sesquilinearity property of the inner product, $\mathcal{V}_{c}^{*}$ is itself a subspace of $\hat{\mathcal{C}}\left[t_{0}, t_{\mathrm{f}}\right]^{n_{\mathbf{u}}} \times \mathbb{R}^{n_{\mathbf{p}}}$. It is 
referred to subsequently as the CS subspace for $\operatorname{OC}\left(\boldsymbol{\theta}^{0}\right)$ at $\left(\mathbf{u}^{*}, \mathbf{p}^{*}\right)$. Moreover, it is self-evident that $\mathcal{V}_{s}^{*} \cap \mathcal{V}_{c}^{*}=\{\mathbf{0}\}$ by construction.

The SS and CS subspaces can thus be seen as properties of the nominal optimal solution $\left(\mathbf{u}^{*}, \mathbf{p}^{*}\right)$ of $\operatorname{OC}(\boldsymbol{\theta})$. The concept of selective input adaptation can now be defined formally.

DeFinition 3.4 (selective input adaptation). The process of adapting the nominal optimal inputs $\left(\mathbf{u}^{*}, \mathbf{p}^{*}\right)$ according to (3.1), (3.2) in any nonzero direction $\left(\boldsymbol{\xi}^{\mathbf{u}}, \boldsymbol{\xi}^{\mathbf{p}}\right) \in$ $\mathcal{V}_{s}^{*}$ is called selective input adaptation along an SS direction. Likewise, the process of adapting $\left(\mathbf{u}^{*}, \mathbf{p}^{*}\right)$ in any nonzero direction $\left(\boldsymbol{\xi}^{\mathbf{u}}, \boldsymbol{\xi}^{\mathbf{p}}\right) \in \mathcal{V}_{c}^{*}$ is called selective input adaptation along a CS direction.

The effect of selective input adaptation along CS and SS directions is analyzed in the following section.

4. Selective input adaptation under parametric uncertainty. Parametric perturbations of the following form are considered throughout this section,

$$
\tilde{\boldsymbol{\theta}}(\eta):=\boldsymbol{\theta}^{0}+\eta \boldsymbol{\xi}^{\boldsymbol{\theta}},
$$

where $\boldsymbol{\xi}^{\boldsymbol{\theta}}$ is a given direction in $\mathbb{R}^{n_{\boldsymbol{\theta}}}$, and the magnitude $\eta$ is such that $|\eta| \ll 1$. For the purpose of this analysis, it is assumed that both $\eta$ and $\boldsymbol{\xi}^{\boldsymbol{\theta}}$ are known.

In the presence of such parametric perturbations, the optimal inputs $\mathbf{u}^{*}, \mathbf{p}^{*}$ are naturally modified. We denote by $\tilde{\mathbf{u}}^{*}(\cdot ; \eta)$ and $\tilde{\mathbf{p}}^{*}(\eta)$ the perturbed optimal inputs, so we have $\tilde{\mathbf{u}}^{*}(\cdot ; 0)=\mathbf{u}^{*}$ and $\tilde{\mathbf{p}}^{*}(0)=\mathbf{p}^{*}$. Failure to apply the perturbed optimal inputs to the process typically leads to a loss of optimality. To characterize this loss, we start by analyzing the variation in cost caused by input adaptation along either SS or CS directions compared to no input adaptation in subsection 4.1. Then, the actual optimality loss with respect to the perturbed optimal control is investigated in subsection 4.2 .

4.1. Cost variation resulting from selective input adaptation. We consider the case that the nominal optimal inputs are adapted according to (3.1), (3.2) along a given direction $\left(\boldsymbol{\xi}^{\mathbf{u}}, \boldsymbol{\xi}^{\mathbf{p}}\right) \in \hat{\mathcal{C}}\left[t_{0}, t_{\mathrm{f}}\right]^{n_{\mathbf{u}}} \times \mathbb{R}^{n_{\mathbf{p}}}$, with a given magnitude $\eta$. Let the corresponding response and cost be denoted by $\tilde{\mathbf{x}}(\cdot ; \eta)$ and $\tilde{J}(\eta)$, respectively. Therefore, we have that $(\tilde{\mathbf{x}}(\cdot ; \eta), \tilde{\mathbf{u}}(\cdot ; \eta), \tilde{\mathbf{p}}(\eta))$ satisfies $(2.2)$ for $\tilde{\boldsymbol{\theta}}$. By the continuous differentiability of $\mathbf{f}$ with respect to $\mathbf{x}, \mathbf{u}$, and $\boldsymbol{\theta}, \tilde{\mathbf{x}}(t ; \eta)$ has a first-order approximation around $\mathbf{x}^{*}(t)$ as

$$
\tilde{\mathbf{x}}(t ; \eta)=\mathbf{x}^{*}(t)+\eta \boldsymbol{\xi}^{\tilde{\mathbf{x}}}(t)+O\left(\eta^{2}\right)
$$

where

$$
\begin{aligned}
\dot{\boldsymbol{\xi}}^{\tilde{\mathbf{x}}}(t) & =\mathbf{f}_{\mathbf{x}}^{*}[t] \boldsymbol{\xi}^{\tilde{\mathbf{x}}}(t)+\mathbf{f}_{\mathbf{u}}^{*}[t] \boldsymbol{\xi}^{\mathbf{u}}(t)+\mathbf{f}_{\mathbf{p}}^{*}[t] \boldsymbol{\xi}^{\mathbf{p}}(t)+\mathbf{f}_{\boldsymbol{\theta}}^{*}[t] \boldsymbol{\xi}^{\boldsymbol{\theta}} \quad \forall t \in\left[t_{k-1}^{*}, t_{k}^{*}\right), k=1, \ldots, N, \\
\boldsymbol{\xi}^{\tilde{\mathbf{x}}}\left(t_{0}\right) & =\mathbf{h}_{\mathbf{p}}^{*} \boldsymbol{\xi}^{\mathbf{p}}+\mathbf{h}_{\boldsymbol{\theta}}^{*} \boldsymbol{\xi}^{\boldsymbol{\theta}}, \quad \boldsymbol{\xi}^{\tilde{\mathbf{x}}}\left(t_{k}^{*+}\right)=\boldsymbol{\xi}^{\tilde{\mathbf{x}}}\left(t_{k}^{*-}\right), \quad k=1, \ldots, N-1 .
\end{aligned}
$$

For comparison purposes, we also consider the case where the nominal optimal inputs $\mathbf{u}^{*}, \mathbf{p}^{*}$ are applied "as is" to the perturbed system. Let the corresponding response and cost be denoted by $\tilde{\mathbf{x}}^{0}(t ; \eta)$ and $\tilde{J}^{0}(\eta)$, respectively. This way, $\left(\tilde{\mathbf{x}}^{0}(\cdot ; \eta), \mathbf{u}^{*}, \mathbf{p}^{*}\right)$ satisfies $(2.2)$ for $\tilde{\boldsymbol{\theta}}$. By the continuous differentiability of $\mathbf{f}$ with respect to $\mathbf{x}$ and $\boldsymbol{\theta}, \tilde{\mathbf{x}}^{0}(t ; \eta)$ has a first-order approximation around $\mathbf{x}^{*}(t)$ given by

$$
\tilde{\mathbf{x}}^{0}(t ; \eta)=\mathbf{x}^{*}(t)+\eta \boldsymbol{\xi}^{0}(t)+O\left(\eta^{2}\right),
$$


where

$$
\begin{aligned}
\dot{\boldsymbol{\xi}}^{\tilde{\mathbf{x}}^{0}}(t) & =\mathbf{f}_{\mathbf{x}}^{*}[t] \tilde{\boldsymbol{\xi}}^{\tilde{\mathbf{x}}^{0}}(t)+\mathbf{f}_{\boldsymbol{\theta}}^{*}[t] \boldsymbol{\xi}^{\boldsymbol{\theta}} \quad \forall t \in\left[t_{k-1}^{*}, t_{k}^{*}\right), \quad k=1, \ldots, N, \\
\boldsymbol{\xi}^{\tilde{\mathbf{x}}^{0}}\left(t_{0}\right) & =\mathbf{h}_{\boldsymbol{\theta}}^{*} \boldsymbol{\xi}^{\boldsymbol{\theta}}, \quad \boldsymbol{\xi}^{\tilde{\mathbf{x}}^{0}}\left(t_{k}^{*+}\right)=\boldsymbol{\xi}^{\tilde{\mathbf{x}}^{0}}\left(t_{k}^{*-}\right), \quad k=1, \ldots, N-1 .
\end{aligned}
$$

While the above two options generally result in suboptimal performance, selective input adaptation can be expected to yield improved performance under a judicious choice of the input adaptation directions. The cost variation due to selective input adaptation is given by

$$
\delta \tilde{J}(\eta):=\tilde{J}(\eta)-\tilde{J}^{0}(\eta) .
$$

The objective of the variational analysis that follows is to compare the effects of input adaptations along any CS direction $\left(\boldsymbol{\xi}_{c}^{\mathbf{u}}, \boldsymbol{\xi}_{c}^{\mathbf{p}}\right) \in \mathcal{V}_{c}^{*}$ and along any SS direction $\left(\boldsymbol{\xi}_{s}^{\mathbf{u}}, \boldsymbol{\xi}_{s}^{\mathbf{p}}\right) \in \mathcal{V}_{s}^{*}$. Note that this analysis will not directly tell us how close the adapted costs are to the perturbed optimal cost; this discussion is deferred until subsection 4.2.

To begin the variational analysis, the cost functionals are first augmented as [5]

$$
\begin{aligned}
J^{a}:= & \varphi\left(t_{\mathrm{f}}, \mathbf{x}\left(t_{\mathrm{f}}\right), \mathbf{p}, \boldsymbol{\theta}\right) \\
& +\sum_{k=1}^{N} \int_{t_{k-1}^{*}}^{t_{k}^{*}}\left[\ell(t, \mathbf{x}(t), \mathbf{u}(t), \mathbf{p}, \boldsymbol{\theta})+\boldsymbol{\pi}(t)^{T}\{\mathbf{f}(t, \mathbf{x}(t), \mathbf{u}(t), \mathbf{p}, \boldsymbol{\theta})-\dot{\mathbf{x}}(t)\}\right] d t
\end{aligned}
$$

for some multiplier functions $\boldsymbol{\pi} \in \hat{\mathcal{C}}^{1}\left[t_{0}, t_{\mathrm{f}}\right]^{n_{\mathrm{x}}}$, where $\hat{\mathcal{C}}^{1}\left[t_{0}, t_{\mathrm{f}}\right]^{n_{\mathrm{x}}}$ represents the linear space of continuous and piecewise continuously differentiable vector-valued functions of dimension $n_{\mathbf{x}}$ on $\left[t_{0}, t_{\mathrm{f}}\right]$. In particular, if the triplet $(\mathbf{x}, \mathbf{u}, \mathbf{p})$ satisfies $(2.2)$ for $\boldsymbol{\theta}$, then $J^{a}=J$ for any $\boldsymbol{\pi} \in \hat{\mathcal{C}}^{1}\left[t_{0}, t_{\mathrm{f}}\right]^{n_{\mathbf{x}}}$, and the problems of minimizing $J$ or $J^{a}$ with respect to $\mathbf{u}, \mathbf{p}$ are equivalent.

Using integration by parts, the expression for $J^{a}$ can be rearranged as follows:

$$
\begin{aligned}
J^{a}= & \varphi\left(t_{\mathrm{f}}, \mathbf{x}\left(t_{\mathrm{f}}\right), \mathbf{p}, \boldsymbol{\theta}\right)+\sum_{k=1}^{N}\left[\boldsymbol{\pi}\left(t_{k-1}^{*}\right)^{T} \mathbf{x}\left(t_{k-1}^{*}\right)-\boldsymbol{\pi}\left(t_{k}^{*}\right)^{T} \mathbf{x}\left(t_{k}^{*}\right)\right] \\
& +\sum_{k=1}^{N} \int_{t_{k-1}^{*}}^{t_{k}^{*}}\left[\ell(t, \mathbf{x}(t), \mathbf{u}(t), \mathbf{p}, \boldsymbol{\theta})+\boldsymbol{\pi}(t)^{T} \mathbf{f}(t, \mathbf{x}(t), \mathbf{u}(t), \mathbf{p}, \boldsymbol{\theta})+\dot{\boldsymbol{\pi}}(t)^{T} \mathbf{x}(t)\right] d t .
\end{aligned}
$$

Since both triplets $(\tilde{\mathbf{x}}(\cdot ; \eta), \tilde{\mathbf{u}}(\cdot ; \eta), \tilde{\mathbf{p}}(\eta))$ and $\left(\tilde{\mathbf{x}}^{0}(\cdot ; \eta), \mathbf{u}^{*}, \mathbf{p}^{*}\right)$ satisfy $(2.2)$ for $\tilde{\boldsymbol{\theta}}$, with respective initial conditions $\tilde{\mathbf{x}}\left(t_{0} ; \eta\right)=\mathbf{h}(\tilde{\mathbf{p}}(\eta), \tilde{\boldsymbol{\theta}})$ and $\tilde{\mathbf{x}}^{0}\left(t_{0} ; \eta\right)=\mathbf{h}\left(\mathbf{p}^{*}, \tilde{\boldsymbol{\theta}}\right)$, the cost variation (4.4) can be written $\operatorname{as}^{3}$

$$
\begin{aligned}
& \delta J(\eta)=\tilde{\varphi}\left[t_{\mathrm{f}}\right]-\tilde{\varphi}^{0}\left[t_{\mathrm{f}}\right]+\boldsymbol{\pi}\left(t_{0}\right)^{T}\left[\tilde{\mathbf{x}}\left(t_{0} ; \eta\right)-\tilde{\mathbf{x}}^{0}\left(t_{0} ; \eta\right)\right] \\
& \quad+\sum_{k=1}^{N}\left(\boldsymbol{\pi}\left(t_{k-1}^{*}\right)^{T}\left[\tilde{\mathbf{x}}\left(t_{k-1}^{*} ; \eta\right)-\tilde{\mathbf{x}}^{0}\left(t_{k-1}^{*} ; \eta\right)\right]-\boldsymbol{\pi}\left(t_{k}^{*}\right)^{T}\left[\tilde{\mathbf{x}}\left(t_{k}^{*} ; \eta\right)-\tilde{\mathbf{x}}^{0}\left(t_{k}^{*} ; \eta\right)\right]\right) \\
& \quad+\sum_{k=1}^{N} \int_{t_{k-1}^{*}}^{t_{k}^{*}}\left(\tilde{\ell}[t]-\tilde{\ell}^{0}[t]+\boldsymbol{\pi}(t)^{T}\left(\tilde{\mathbf{f}}[t]-\tilde{\mathbf{f}}^{0}[t]\right)+\dot{\boldsymbol{\pi}}(t)^{T}\left[\tilde{\mathbf{x}}(t ; \eta)-\tilde{\mathbf{x}}^{0}(t ; \eta)\right]\right) d t .
\end{aligned}
$$

\footnotetext{
${ }^{3}$ The additional compact notation $y^{0}[t] \quad:=y\left(t, \tilde{\mathbf{x}}^{0}(t ; \eta), \mathbf{u}^{*}(t), \mathbf{p}^{*}, \tilde{\boldsymbol{\theta}}(\eta)\right)$ and $\tilde{y}[t] \quad:=$ $y(t, \tilde{\mathbf{x}}(t ; \eta), \tilde{\mathbf{u}}(t ; \eta), \tilde{\mathbf{p}}(\eta), \tilde{\boldsymbol{\theta}}(\eta))$ are used in the remainder of the paper.
} 
Taylor expanding $\tilde{\varphi}\left[t_{\mathrm{f}}\right], \tilde{\ell}[t], \tilde{\mathbf{f}}[t]$, and $\tilde{\mathbf{h}}$ around $\left(\tilde{\mathbf{x}}^{0}(t ; \eta), \mathbf{u}^{*}(t), \mathbf{p}^{*}, \tilde{\boldsymbol{\theta}}(\eta)\right)$ and rearranging the terms in (4.5) using (4.3) and (4.2) gives

$$
\begin{aligned}
& \delta J(\eta)=\eta\left(\left[\tilde{\varphi}_{\mathbf{x}}^{0}\left[t_{\mathrm{f}}\right]^{T}-\boldsymbol{\pi}\left(t_{\mathrm{f}}\right)^{T}\right]\left[\boldsymbol{\xi}^{\tilde{\mathbf{x}}}\left(t_{\mathrm{f}}\right)-\boldsymbol{\xi}^{\tilde{\mathbf{x}}^{0}}\left(t_{\mathrm{f}}\right)\right]+\tilde{\varphi}_{\mathbf{p}}^{0}\left[t_{\mathrm{f}}\right]^{T} \boldsymbol{\xi}^{\mathbf{p}}+\boldsymbol{\pi}\left(t_{0}\right)^{T} \tilde{\mathbf{h}}_{\mathbf{p}}^{0} \boldsymbol{\xi}^{\mathbf{p}}\right. \\
& \quad+\sum_{k=1}^{N} \int_{t_{k-1}^{*}}^{t_{k}^{*}}\left[\tilde{\ell}_{\mathbf{x}}^{0}[t]^{T}+\boldsymbol{\pi}(t)^{T} \tilde{\mathbf{f}}_{\mathbf{x}}^{0}[t]+\dot{\boldsymbol{\pi}}(t)^{T}\right]\left[\boldsymbol{\xi}^{\tilde{\mathbf{x}}}(t)-\boldsymbol{\xi}^{\tilde{\mathbf{x}}^{0}}(t)\right] d t \\
& \left.\quad+\sum_{k=1}^{N} \int_{t_{k-1}^{*}}^{t_{k}^{*}}\left[\left(\tilde{\ell}_{\mathbf{u}}^{0}[t]^{T}+\boldsymbol{\pi}(t)^{T} \tilde{\mathbf{f}}_{\mathbf{u}}^{0}[t]\right) \boldsymbol{\xi}^{\mathbf{u}}(t)+\left(\tilde{\ell}_{\mathbf{p}}^{0}[t]^{T}+\boldsymbol{\pi}(t)^{T} \tilde{\mathbf{f}}_{\mathbf{p}}^{0}[t]\right) \boldsymbol{\xi}^{\mathbf{p}}\right] d t\right)+O\left(\eta^{2}\right) .
\end{aligned}
$$

Since the multiplier functions $\boldsymbol{\pi}(t)$ are arbitrary, they can be specialized as the (unique) solution $\tilde{\boldsymbol{\pi}}^{0}$ to the linear system

$$
\begin{aligned}
\dot{\tilde{\pi}}^{0}(t ; \eta) & =-\tilde{\mathbf{f}}_{\mathbf{x}}^{0}[t]^{T} \tilde{\boldsymbol{\pi}}^{0}(t ; \eta)-\tilde{\ell}_{\mathbf{x}}^{0}[t] \quad \forall t \in\left(t_{k-1}^{*}, t_{k}^{*}\right], k=1, \ldots, N, \\
\tilde{\boldsymbol{\pi}}^{0}\left(t_{\mathrm{f}} ; \eta\right) & =\tilde{\varphi}_{\mathbf{x}}^{0}\left[t_{\mathrm{f}}\right], \quad \tilde{\boldsymbol{\pi}}^{0}\left(t_{k}^{*-} ; \eta\right)=\tilde{\boldsymbol{\pi}}^{0}\left(t_{k}^{*+} ; \eta\right), \quad k=1, \ldots, N-1 .
\end{aligned}
$$

This way, and after Taylor expanding the terms $\tilde{\ell}_{\mathbf{u}}^{0}[t], \tilde{\mathbf{f}}_{\mathbf{x}}^{0}[t]$, and $\tilde{\mathbf{f}}_{\mathbf{u}}^{0}[t]$ around $\left(\mathbf{x}^{*}(t)\right.$, $\left.\mathbf{u}^{*}(t), \mathbf{p}^{*}, \boldsymbol{\theta}^{0}\right)$, the cost variation reduces to

$$
\begin{aligned}
& \delta J(\eta)=\eta\left(\sum_{k=1}^{N} \int_{t_{k-1}^{*}}^{t_{k}^{*}}\left(\ell_{\mathbf{u}}^{*}[t]^{T}+\tilde{\boldsymbol{\pi}}^{0}(t ; \eta)^{T} \mathbf{f}_{\mathbf{u}}^{*}[t]\right) \boldsymbol{\xi}^{\mathbf{u}}(t) d t\right. \\
& \left.\quad+\left[\varphi_{\mathbf{p}}^{*}\left[t_{\mathrm{f}}\right]^{T}+\tilde{\boldsymbol{\pi}}^{0}\left(t_{0} ; \eta\right)^{T} \mathbf{h}_{\mathbf{p}}^{*}+\sum_{k=1}^{N} \int_{t_{k-1}^{*}}^{t_{k}^{*}}\left(\ell_{\mathbf{p}}^{*}[t]^{T}+\tilde{\boldsymbol{\pi}}^{0}(t ; \eta)^{T} \mathbf{f}_{\mathbf{p}}^{*}[t]\right) d t\right] \boldsymbol{\xi}^{\mathbf{p}}\right)+O\left(\eta^{2}\right) .
\end{aligned}
$$

Since the optimality conditions (2.5), (2.6) hold along the nominal optimal solution, (4.8) can be rewritten as

$$
\begin{aligned}
& \delta J(\eta)=\eta\left(\sum_{k=1}^{N} \int_{t_{k-1}^{*}}^{t_{k}^{*}}\left(\left[\tilde{\boldsymbol{\pi}}^{0}(t ; \eta)-\boldsymbol{\lambda}^{*}(t)\right]^{T} \mathbf{f}_{\mathbf{u}}^{*}[t]-\boldsymbol{\mu}^{a}(t)^{T} \boldsymbol{\Omega}_{\mathbf{u}}^{a}[t]\right) \boldsymbol{\xi}^{\mathbf{u}}(t) d t\right. \\
& \quad+\boldsymbol{\rho}^{a T} \mathbf{T}_{\mathbf{p}}^{a}\left[t_{\mathrm{f}}\right] \boldsymbol{\xi}^{\mathbf{p}}+\left[\tilde{\boldsymbol{\pi}}^{0}\left(t_{0} ; \eta\right)-\boldsymbol{\lambda}^{*}\left(t_{0}\right)\right]^{T} \mathbf{h}_{\mathbf{p}}^{*} \boldsymbol{\xi}^{\mathbf{p}} \\
& \left.\quad+\sum_{k=1}^{N} \int_{t_{k-1}^{*}}^{t_{k}^{*}}\left(\left[\tilde{\boldsymbol{\pi}}^{0}(t ; \eta)-\boldsymbol{\lambda}^{*}(t)\right]^{T} \mathbf{f}_{\mathbf{p}}^{*}[t]-\boldsymbol{\mu}^{a}(t)^{T} \boldsymbol{\Omega}_{\mathbf{p}}^{a}[t]\right) \boldsymbol{\xi}^{\mathbf{p}} d t\right)+O\left(\eta^{2}\right) .
\end{aligned}
$$

Let $\boldsymbol{\pi}^{*}(t)$ be the (unique) solution to the linear system

$$
\begin{aligned}
\dot{\boldsymbol{\pi}}^{*}(t) & =-\mathbf{f}_{\mathbf{x}}^{*}[t]^{T} \boldsymbol{\pi}^{*}(t)-\ell_{\mathbf{x}}^{*}[t] \quad \forall t \in\left(t_{k-1}^{*}, t_{k}^{*}\right], k=1, \ldots, N, \\
\boldsymbol{\pi}^{*}\left(t_{\mathrm{f}}\right) & =\varphi_{\mathbf{x}}^{*}\left[t_{\mathrm{f}}\right], \quad \boldsymbol{\pi}^{*}\left(t_{k}^{*-}\right)=\boldsymbol{\pi}^{*}\left(t_{k}^{*+}\right), \quad k=1, \ldots, N-1 .
\end{aligned}
$$

Using (4.7) and (4.10), it should be clear that

$$
\tilde{\boldsymbol{\pi}}^{0}(t ; \eta)=\boldsymbol{\pi}^{*}(t)+O(\eta)
$$

The term $\left[\tilde{\boldsymbol{\pi}}^{0}(t ; \eta)-\boldsymbol{\lambda}^{*}(t)\right]$ in (4.9) can thus be rewritten as

$$
\tilde{\boldsymbol{\pi}}^{0}(t ; \eta)-\boldsymbol{\lambda}^{*}(t)=\boldsymbol{\beta}^{*}(t)+O(\eta),
$$


where $\boldsymbol{\beta}^{*}(t)=\boldsymbol{\pi}^{*}(t)-\boldsymbol{\lambda}^{*}(t)$ satisfies

$$
\begin{aligned}
\dot{\boldsymbol{\beta}}^{*}(t) & =-\mathbf{f}_{\mathbf{x}}^{*}[t]^{T} \boldsymbol{\beta}^{*}(t)+\boldsymbol{\Omega}_{\mathbf{x}}^{a}[t]^{T} \boldsymbol{\mu}^{a}(t) \quad \forall t \in\left(t_{k-1}^{*}, t_{k}^{*}\right], k=1, \ldots, N, \\
\boldsymbol{\beta}^{*}\left(t_{\mathrm{f}}\right) & =-\mathbf{T}_{\mathbf{x}}^{a}\left[t_{\mathrm{f}}\right]^{T} \boldsymbol{\rho}^{a}, \quad \boldsymbol{\beta}^{*}\left(t_{k}^{*-}\right)=\boldsymbol{\beta}^{*}\left(t_{k}^{*+}\right), \quad k=1, \ldots, N-1,
\end{aligned}
$$

since $\boldsymbol{\lambda}^{*}(t)$ satisfies (2.7). The linear dynamic model for $\boldsymbol{\beta}^{*}(t)$ has the following unique solution on $\left[t_{0}, t_{\mathrm{f}}\right]$ :

$$
\begin{aligned}
\boldsymbol{\beta}^{*}(t)= & -\boldsymbol{\Phi}^{\mathbf{f}_{\mathbf{x}}^{*}}\left(t_{\mathrm{f}}, t\right)^{T} \mathbf{T}_{\mathbf{x}}^{a}\left[t_{\mathrm{f}}\right]^{T} \boldsymbol{\rho}^{a}-\int_{t}^{t_{k}^{*}} \boldsymbol{\Phi}^{\mathbf{f}_{\mathbf{x}}^{*}}(s, t)^{T} \boldsymbol{\Omega}_{\mathbf{x}}^{a}[s]^{T} \boldsymbol{\mu}^{a}(s) d s \\
& -\sum_{i=k+1}^{N} \int_{t_{i-1}^{*}}^{t_{i}^{*}} \boldsymbol{\Phi}^{\mathbf{f}_{\mathbf{x}}^{*}}(s, t)^{T} \boldsymbol{\Omega}_{\mathbf{x}}^{a}[s]^{T} \boldsymbol{\mu}^{a}(s) d s
\end{aligned}
$$

for each $t \in\left(t_{k-1}^{*}, t_{k}^{*}\right], k=1, \ldots, N$. Combining (4.12) and (4.9) gives

$$
\begin{aligned}
& \delta J(\eta)=-\eta \sum_{k=1}^{N} \int_{t_{k-1}^{*}}^{t_{k}^{*}}\left[\boldsymbol{\mu}^{a}(t)^{T}\left(\boldsymbol{\Omega}_{\mathbf{u}}^{a}[t] \boldsymbol{\xi}^{\mathbf{u}}(t)+\boldsymbol{\Omega}_{\mathbf{p}}^{a}[t] \boldsymbol{\xi}^{\mathbf{p}}\right)\right. \\
& +\int_{t}^{t_{k}^{*}} \boldsymbol{\mu}^{a}(s)^{T} \boldsymbol{\Omega}_{\mathbf{x}}^{a}[s] \mathbf{\Phi}^{\mathbf{f}_{\mathbf{x}}^{*}}(s, t)\left(\mathbf{f}_{\mathbf{u}}^{*}[t] \boldsymbol{\xi}^{\mathbf{u}}(t)+\mathbf{f}_{\mathbf{p}}^{*}[t] \boldsymbol{\xi}^{\mathbf{p}}\right) d s \\
& \left.+\sum_{i=k+1}^{N} \int_{t_{i-1}^{*}}^{t_{i}^{*}} \boldsymbol{\mu}^{a}(s)^{T} \boldsymbol{\Omega}_{\mathbf{x}}^{a}[s] \Phi^{\mathbf{f}_{\mathbf{x}}^{*}}(s, t)\left(\mathbf{f}_{\mathbf{u}}^{*}[t] \boldsymbol{\xi}^{\mathbf{u}}(t)+\mathbf{f}_{\mathbf{p}}^{*}[t] \boldsymbol{\xi}^{\mathbf{p}}\right) d s\right] d t \\
& -\eta \boldsymbol{\rho}^{a T} \mathbf{T}_{\mathbf{x}}^{a}\left[t_{\mathrm{f}}\right] \sum_{k=1}^{N} \int_{t_{k-1}^{*}}^{t_{k}^{*}} \boldsymbol{\Phi}^{\mathbf{f}_{\mathbf{x}}^{*}}\left(t_{\mathrm{f}}, t\right)\left(\mathbf{f}_{\mathbf{u}}^{*}[t] \boldsymbol{\xi}^{\mathbf{u}}(t)+\mathbf{f}_{\mathbf{p}}^{*}[t] \boldsymbol{\xi}^{\mathbf{p}}\right) d t-\eta \boldsymbol{\rho}^{a T} \mathbf{T}_{\mathbf{p}}^{a}\left[t_{\mathrm{f}}\right] \boldsymbol{\xi}^{\mathbf{p}} \\
& -\eta\left[\boldsymbol{\rho}^{a T} \mathbf{T}_{\mathbf{x}}^{a}\left[t_{\mathrm{f}}\right] \boldsymbol{\Phi}^{\mathbf{f}_{\mathbf{x}}^{*}}\left(t_{\mathrm{f}}, t_{0}\right)+\sum_{k=1}^{N} \int_{t_{k-1}^{*}}^{t_{k}^{*}} \boldsymbol{\mu}^{a}(t)^{T} \boldsymbol{\Omega}_{\mathbf{x}}^{a}[t] \mathbf{\Phi}^{\mathbf{f}_{\mathbf{x}}^{*}}\left(t, t_{0}\right) d t\right] \mathbf{h}_{\mathbf{p}}^{*} \boldsymbol{\xi}^{\mathbf{p}}+O\left(\eta^{2}\right) .
\end{aligned}
$$

Using (3.12), the next-to-last line in (4.13), together with the first term in the last line, is equal to $-\eta \boldsymbol{\rho}^{a T} \mathcal{D}_{\mathbf{T}^{a}, t_{\mathbf{f}}}\left(\boldsymbol{\xi}^{\mathbf{u}}, \boldsymbol{\xi}^{\mathbf{p}}\right)$. Furthermore, the order of integration in all double integral terms in (4.13) can be changed as follows [13]:

$$
\begin{aligned}
\int_{t_{k-1}^{*}}^{t_{k}^{*}} & {\left[\int_{t}^{t_{k}^{*}} \boldsymbol{\mu}^{a}(s)^{T} \boldsymbol{\Omega}_{\mathbf{x}}^{a}[s] \mathbf{\Phi}^{\mathbf{f}_{\mathbf{x}}^{*}}(s, t)\left(\mathbf{f}_{\mathbf{u}}^{*}[t] \boldsymbol{\xi}^{\mathbf{u}}(t)+\mathbf{f}_{\mathbf{p}}^{*}[t] \boldsymbol{\xi}^{\mathbf{p}}\right) d s\right.} \\
& \left.+\sum_{i=k+1}^{N} \int_{t_{i-1}^{*}}^{t_{i}^{*}} \boldsymbol{\mu}^{a}(s)^{T} \boldsymbol{\Omega}_{\mathbf{x}}^{a}[s] \mathbf{\Phi}_{\mathbf{x}}^{\mathbf{f}_{\mathbf{x}}^{*}}(s, t)\left(\mathbf{f}_{\mathbf{u}}^{*}[t] \boldsymbol{\xi}^{\mathbf{u}}(t)+\mathbf{f}_{\mathbf{p}}^{*}[t] \boldsymbol{\xi}^{\mathbf{p}}\right) d s\right] d t \\
= & \int_{t_{k-1}^{*}}^{t_{k}^{*}} \boldsymbol{\mu}^{a}(s)^{T} \boldsymbol{\Omega}_{\mathbf{x}}^{a}[s]\left[\int_{t_{k-1}^{*}}^{t} \boldsymbol{\Phi}_{\mathbf{x}}^{\mathbf{f}_{\mathbf{x}}^{*}}(s, t)\left(\mathbf{f}_{\mathbf{u}}^{*}[t] \boldsymbol{\xi}^{\mathbf{u}}(t)+\mathbf{f}_{\mathbf{p}}^{*}[t] \boldsymbol{\xi}^{\mathbf{p}}\right) d t\right] d s \\
& +\sum_{i=k+1}^{N} \int_{t_{i-1}^{*}}^{t_{i}^{*}} \boldsymbol{\mu}^{a}(s)^{T} \boldsymbol{\Omega}_{\mathbf{x}}^{a}[s]\left[\int_{t_{k-1}^{*}}^{t_{k}^{*}} \boldsymbol{\Phi}^{\mathbf{f}_{\mathbf{x}}^{*}}(s, t)\left(\mathbf{f}_{\mathbf{u}}^{*}[t] \boldsymbol{\xi}^{\mathbf{u}}(t)+\mathbf{f}_{\mathbf{p}}^{*}[t] \boldsymbol{\xi}^{\mathbf{p}}\right) d t\right] d s .
\end{aligned}
$$

Noting that

$$
\sum_{k=1}^{N} \sum_{i=k+1}^{N} \alpha_{i, k}=\sum_{i=1}^{N} \sum_{k=1}^{i-1} \alpha_{i, k}
$$


the double integral terms in (4.13) yield

$$
\begin{aligned}
& \sum_{k=1}^{N} \int_{t_{k-1}^{*}}^{t_{k}^{*}}\left[\int_{t}^{t_{k}^{*}} \boldsymbol{\mu}^{a}(s)^{T} \boldsymbol{\Omega}_{\mathbf{x}}^{a}[s] \boldsymbol{\Phi}^{\mathbf{f}_{\times}^{*}}(s, t)\left(\mathbf{f}_{\mathbf{u}}^{*}[t] \boldsymbol{\xi}^{\mathbf{u}}(t)+\mathbf{f}_{\mathbf{p}}^{*}[t] \boldsymbol{\xi}^{\mathbf{p}}\right) d s\right. \\
& \left.+\sum_{i=k+1}^{N} \int_{t_{i-1}^{*}}^{t_{i}^{*}} \boldsymbol{\mu}^{a}(s)^{T} \boldsymbol{\Omega}_{\mathbf{x}}^{a}[s] \boldsymbol{\Phi}^{\mathbf{f}_{\mathbf{f}}^{*}}(s, t)\left(\mathbf{f}_{\mathbf{u}}^{*}[t] \boldsymbol{\xi}^{\mathbf{u}}(t)+\mathbf{f}_{\mathbf{p}}^{*}[t] \boldsymbol{\xi}^{\mathbf{p}}\right) d s\right] d t \\
& =\sum_{k=1}^{N} \int_{t_{k-1}^{*}}^{t_{k}^{*}} \boldsymbol{\mu}^{a}(s)^{T} \boldsymbol{\Omega}_{\mathbf{x}}^{a}[s]\left[\int_{t_{k-1}^{*}}^{t} \boldsymbol{\Phi}^{\mathbf{f}_{\mathbf{x}}^{*}}(s, t) \mathbf{f}_{\mathbf{u}}^{*}[t] \boldsymbol{\xi}^{\mathbf{u}}(t) d t\right] d s \\
& +\sum_{i=1}^{N} \int_{t_{i-1}^{*}}^{t_{i}^{*}} \boldsymbol{\mu}^{a}(s)^{T} \boldsymbol{\Omega}_{\mathbf{x}}^{a}[s]\left[\sum_{k=1}^{i-1} \int_{t_{k-1}^{*}}^{t_{k}^{*}} \boldsymbol{\Phi}^{\mathbf{f}_{\mathbf{x}}^{*}}(s, t)\left(\mathbf{f}_{\mathbf{u}}^{*}[t] \boldsymbol{\xi}^{\mathbf{u}}(t)+\mathbf{f}_{\mathbf{p}}^{*}[t] \boldsymbol{\xi}^{\mathbf{p}}\right) d t\right] d s \\
& =\sum_{k=1}^{N} \int_{t_{k-1}^{*}}^{t_{k}^{*}} \boldsymbol{\mu}^{a}(s)^{T} \boldsymbol{\Omega}_{\mathbf{x}}^{a}[s]\left[\sum_{i=1}^{k-1} \int_{t_{i-1}^{*}}^{t_{i}^{*}} \boldsymbol{\Phi}^{\mathbf{f}_{\times}^{*}}(s, t)\left(\mathbf{f}_{\mathbf{u}}^{*}[t] \boldsymbol{\xi}^{\mathbf{u}}(t)+\mathbf{f}_{\mathbf{p}}^{*}[t] \boldsymbol{\xi}^{\mathbf{p}}\right) d t\right. \\
& \left.+\int_{t_{k-1}^{*}}^{t} \boldsymbol{\Phi}^{\mathbf{f}_{\times}^{*}}(s, t)\left(\mathbf{f}_{\mathbf{u}}^{*}[t] \boldsymbol{\xi}^{\mathbf{u}}(t)+\mathbf{f}_{\mathbf{p}}^{*}[t] \boldsymbol{\xi}^{\mathbf{p}}\right) d t\right] d s .
\end{aligned}
$$

Using (3.13), it follows that the first term in (4.13), together with the second term in the last line, is equal to $-\eta \sum_{k=1}^{N} \int_{t_{k-1}^{*}}^{t_{*}^{*}} \boldsymbol{\mu}^{a}(t)^{T} \mathcal{D}_{\boldsymbol{\Omega}^{a}, t}\left(\boldsymbol{\xi}^{\mathbf{u}}, \boldsymbol{\xi}^{\mathbf{p}}\right) d t$. Finally, the expression (4.13) of the cost variation can be rewritten as

$$
\delta J(\eta)=-\eta\left[\boldsymbol{\rho}^{a T} \mathcal{D}_{\mathbf{T}^{a}, t_{\mathrm{f}}}\left(\boldsymbol{\xi}^{\mathbf{u}}, \boldsymbol{\xi}^{\mathbf{p}}\right)+\sum_{k=1}^{N} \int_{t_{k-1}^{*}}^{t_{k}^{*}} \boldsymbol{\mu}^{a}(t)^{T} \mathcal{D}_{\boldsymbol{\Omega}^{a}, t}\left(\boldsymbol{\xi}^{\mathbf{u}}, \boldsymbol{\xi}^{\mathbf{p}}\right) d t\right]+O\left(\eta^{2}\right) .
$$

We are now ready to state the main result of the paper.

THEOREM 4.1 (cost variation resulting from selective input adaptation). Let $\left(\mathbf{u}^{*}, \mathbf{p}^{*}\right)$ be an optimal solution for the optimal control problem $O C\left(\boldsymbol{\theta}^{0}\right)$, and consider parametric perturbations of the form (4.1). The cost variation $\delta J(\eta)$ resulting from selective input adaptation of type (3.1), (3.2) along any (nonzero) SS direction $\left(\boldsymbol{\xi}_{s}^{\mathbf{u}}, \boldsymbol{\xi}_{s}^{\mathbf{p}}\right) \in \mathcal{V}_{s}^{*}$ is $O\left(\eta^{2}\right)$, whereas the cost variation resulting from adaptation along any (nonzero) CS direction $\left(\boldsymbol{\xi}_{c}^{\mathbf{u}}, \boldsymbol{\xi}_{c}^{\mathbf{p}}\right) \in \mathcal{V}_{c}^{*}$ is $O(\eta)$.

Proof. By Definition 3.2, any direction $\left(\boldsymbol{\xi}_{s}^{\mathbf{u}}, \boldsymbol{\xi}_{s}^{\mathbf{p}}\right) \in \mathcal{V}_{s}^{*}$ satisfies the system of linear integral equations (3.12) and (3.13). Therefore, from (4.14), $\delta J(\eta)=O\left(\eta^{2}\right)$. On the other hand, no nonzero direction $\left(\boldsymbol{\xi}_{c}^{\mathbf{u}}, \boldsymbol{\xi}_{c}^{\mathbf{p}}\right) \in \mathcal{V}_{c}^{*}$ satisfies (3.12) and (3.13) by construction. Since strict complementarity slackness holds for the terminal and path constraints at $\left(\mathbf{u}^{*}, \mathbf{p}^{*}\right)$, the first-order term in (4.14) is nonzero in general, and it follows that $\delta J(\eta)=O(\eta)$.

The main implication of Theorem 4.1 is that, for small parametric perturbations, adapting the inputs along CS directions has the largest impact on the performance of the perturbed system, while the effect of not adapting the inputs along SS directions is relatively smaller. Accordingly, when designing a practical input adaptation strategy for problem $\mathrm{OC}(\tilde{\boldsymbol{\theta}})$, priority should be given to meeting the active terminal and path constraints (2.3), (2.4) over enforcing the Hamiltonian sensitivity condition (2.5), (2.6). It should also be noted that, while a judicious choice of adaptation direction $\left(\boldsymbol{\xi}_{c}^{\mathbf{u}}, \boldsymbol{\xi}_{c}^{\mathbf{p}}\right) \in \mathcal{V}_{c}^{*}$ will lead to substantial performance improvement, a poor choice of 
$\left(\boldsymbol{\xi}_{c}^{\mathbf{u}}, \boldsymbol{\xi}_{c}^{\mathbf{p}}\right)$ will be detrimental to the performance of the adapted system. The implications of selective input adaptation on reducing the optimality gap due to parametric perturbations are discussed in the following subsection.

4.2. Implications of selective input adaptation on optimality loss. The variational analysis in subsection 4.1 considers the effect of a selective input adaptation with respect to the nominal operating conditions. However, it does not consider the effect in terms of the actual optimality loss,

$$
\delta \tilde{J}^{*}(\eta):=\tilde{J}^{*}(\eta)-\tilde{J}(\eta),
$$

where $\tilde{J}^{*}(\eta)$ stands for the optimal perturbed cost, which corresponds to the optimal inputs $\tilde{\mathbf{u}}^{*}(\cdot ; \eta), \tilde{\mathbf{p}}^{*}(\eta)$ and the corresponding optimal response $\tilde{\mathbf{x}}^{*}(\cdot ; \eta)$ in the presence of parametric uncertainty (4.1).

To conduct this analysis, we make the following additional assumptions:

i. The sets of active terminal and path constraints in the perturbed optimal solution remain the same as in the nominal optimal solution, and

ii. the optimal inputs are continuously differentiable with respect to the parametric perturbation (4.1) in a neighborhood of $\eta=0$.

Sufficient conditions under which these assumptions hold have been discussed extensively in the literature, e.g., [21, 29, 26, 28, 22]; see also [10] for a derivation, based on the theory of neighboring extremals $[5,30]$, of the first-order variations of the optimal inputs with respect to parametric perturbations in optimal control problems with mixed control-state constraints.

Under these assumptions, $\tilde{\mathbf{u}}^{*}(t ; \eta)$ and $\tilde{\mathbf{p}}^{*}(\eta)$ have first-order approximations around $\mathbf{u}^{*}(t)$ and $\mathbf{p}^{*}$ as

$$
\begin{aligned}
\tilde{\mathbf{u}}^{*}(t ; \eta) & =\mathbf{u}^{*}(t)+\eta \boldsymbol{\xi}^{\tilde{\mathbf{u}}, *}(t)+O\left(\eta^{2}\right), \\
\tilde{\mathbf{p}}^{*}(\eta) & =\mathbf{p}^{*}+\eta \boldsymbol{\xi}^{\tilde{\mathbf{p}}, *}+O\left(\eta^{2}\right)
\end{aligned}
$$

for some $\left(\boldsymbol{\xi}^{\tilde{\mathbf{u}}, *}, \boldsymbol{\xi}^{\tilde{\mathbf{p}}, *}\right) \in \hat{\mathcal{C}}\left[t_{0}, t_{\mathrm{f}}\right]^{n_{\mathbf{u}}} \times \mathbb{R}^{n_{\mathbf{p}}}$. And by differentiability of $\mathbf{f}$ with respect to $\mathbf{x}, \mathbf{u}$, and $\boldsymbol{\theta}$, it follows that $\tilde{\mathbf{x}}^{*}(t ; \eta)$ also has first-order approximations around $\mathbf{x}^{*}(t)$ as

$$
\tilde{\mathbf{x}}^{*}(t ; \eta)=\mathbf{x}^{*}(t)+\eta \boldsymbol{\xi}^{\tilde{\mathbf{x}}, *}(t)+O\left(\eta^{2}\right) .
$$

Consider the active terminal constraints first. Since $\mathbf{T}^{a}\left(t_{\mathrm{f}}, \tilde{\mathbf{x}}^{*}\left(t_{\mathrm{f}} ; \eta\right), \tilde{\mathbf{p}}^{*}(\eta), \tilde{\boldsymbol{\theta}}(\eta)\right)=$ $\mathbf{0}$ in a neighborhood of $\eta=0$, it follows that

$$
\mathbf{T}_{\mathbf{x}}^{a}\left[t_{\mathrm{f}}\right] \boldsymbol{\xi}^{\tilde{\mathbf{x}}, *}\left(t_{\mathrm{f}}\right)+\mathbf{T}_{\mathbf{u}}^{a}\left[t_{\mathrm{f}}\right] \boldsymbol{\xi}^{\tilde{\mathbf{u}}, *}\left(t_{\mathrm{f}}\right)+\mathbf{T}_{\mathbf{p}}^{a}\left[t_{\mathrm{f}}\right] \boldsymbol{\xi}^{\tilde{\mathbf{p}}, *}+\mathbf{T}_{\boldsymbol{\theta}}^{a}\left[t_{\mathrm{f}}\right] \boldsymbol{\xi}^{\boldsymbol{\theta}}=\mathbf{0} .
$$

By a similar argument as in subsection 3.2, it is then established that

$$
\mathcal{D}_{\mathbf{T}^{a}, t_{\mathrm{f}}}^{*}\left(\boldsymbol{\xi}^{\tilde{\mathbf{u}}, *}, \boldsymbol{\xi}^{\tilde{\mathbf{p}}, *}\right)+\mathcal{O}_{\mathbf{T}^{a}, t_{\mathrm{f}}}^{*}\left(\boldsymbol{\xi}^{\boldsymbol{\theta}}\right)=\mathbf{0},
$$

where the linear operator $\mathcal{O}_{\Psi, \underline{t}}^{*}: \mathbb{R}^{n_{\boldsymbol{\theta}}} \rightarrow \mathbb{R}^{n_{\psi}}$ is defined as

$$
\begin{gathered}
\mathcal{O}_{\boldsymbol{\Psi}, \underline{t}}^{*}\left(\boldsymbol{\xi}^{\boldsymbol{\theta}}\right):=\boldsymbol{\Psi}_{\mathbf{\times}}^{*}[\underline{t}]\left[\boldsymbol{\Phi}_{\times}^{\mathbf{f}_{\times}^{*}}\left(\underline{t}, t_{0}\right) \mathbf{h}_{\boldsymbol{\theta}}^{*} \boldsymbol{\xi}^{\boldsymbol{\theta}}+\sum_{i=1}^{k-1} \int_{t_{i-1}^{*}}^{t_{i}^{*}} \boldsymbol{\Phi}^{\mathbf{f}_{\times}^{*}}(\underline{t}, s) \mathbf{f}_{\boldsymbol{\theta}}^{*}[s] \boldsymbol{\xi}^{\boldsymbol{\theta}} d s\right. \\
\left.+\int_{t_{k-1}^{*}}^{\underline{t}} \boldsymbol{\Phi}^{\mathbf{f}_{\times}^{*}}(\underline{t}, s) \mathbf{f}_{\boldsymbol{\theta}}^{*}[s] \boldsymbol{\xi}^{\boldsymbol{\theta}} d s\right]+\boldsymbol{\Psi}_{\boldsymbol{\theta}}^{*}[\underline{t}] \boldsymbol{\xi}^{\boldsymbol{\theta}} .
\end{gathered}
$$


In the case of active path constraints, a similar argument shows that

$$
\mathcal{D}_{\boldsymbol{\Omega}^{a}, t}^{*}\left(\boldsymbol{\xi}^{\tilde{\mathbf{u}}, *}, \boldsymbol{\xi}^{\tilde{\mathbf{p}}, *}\right)+\mathcal{O}_{\boldsymbol{\Omega}^{a}, t}^{*}\left(\boldsymbol{\xi}^{\boldsymbol{\theta}}\right)=\mathbf{0} \quad \forall t \in\left(t_{k-1}^{*}, t_{k}^{*}\right], \quad k=1, \ldots, N .
$$

Using (4.14), (4.19), and (4.20), the optimality loss $\delta \tilde{J}^{*}(\eta)$ in (4.15) can therefore be rewritten as

$$
\delta \tilde{J}^{*}(\eta)=\eta\left[\boldsymbol{\rho}^{a T} \mathcal{O}_{\mathbf{T}^{a}, t_{\mathrm{f}}} \boldsymbol{\xi}^{\boldsymbol{\theta}}+\sum_{k=1}^{N} \int_{t_{k-1}^{*}}^{t_{k}^{*}} \boldsymbol{\mu}^{a}(t)^{T} \mathcal{O}_{\boldsymbol{\Omega}^{a}, t} \boldsymbol{\xi}^{\boldsymbol{\theta}} d t\right]+O\left(\eta^{2}\right) .
$$

These considerations lead to the following corollary of Theorem 4.1.

COROLLARY 4.2 (optimality gap resulting from selective input adaptation). No selective input adaptation along an SS direction $\left(\boldsymbol{\xi}_{s}^{\mathbf{u}}, \boldsymbol{\xi}_{s}^{\mathbf{p}}\right) \in \mathcal{V}_{s}^{*}$ is able to close the firstorder optimality loss term in (4.21). Only adaptation along a CS direction $\left(\boldsymbol{\xi}_{c}^{\mathbf{u}}, \boldsymbol{\xi}_{c}^{\mathbf{p}}\right) \in$ $\mathcal{V}_{c}^{*}$ is able to reduce this loss. In particular, any adaptation direction $\left(\boldsymbol{\xi}^{\mathbf{u}}, \boldsymbol{\xi}^{\mathbf{p}}\right) \in$ $\hat{\mathcal{C}}\left[t_{0}, t_{\mathrm{f}}\right]^{n_{\mathbf{u}}} \times \mathbb{R}^{n_{\mathbf{p}}}$ such that

$$
\begin{aligned}
\mathcal{D}_{\mathbf{T}^{a}, t_{\mathrm{f}}}^{*}\left(\boldsymbol{\xi}^{\mathbf{u}}, \boldsymbol{\xi}^{\mathbf{p}}\right)+\mathcal{O}_{\mathbf{T}^{a}, t_{\mathrm{f}}}^{*}\left(\boldsymbol{\xi}^{\boldsymbol{\theta}}\right) & =\mathbf{0}, \\
\mathcal{D}_{\boldsymbol{\Omega}^{a}, t}^{*}\left(\boldsymbol{\xi}^{\mathbf{u}}, \boldsymbol{\xi}^{\mathbf{p}}\right)+\mathcal{O}_{\boldsymbol{\Omega}^{a}, t}^{*}\left(\boldsymbol{\xi}^{\boldsymbol{\theta}}\right) & =\mathbf{0} \quad \forall t \in\left(t_{k-1}^{*}, t_{k}^{*}\right], k=1, \ldots, N,
\end{aligned}
$$

will cancel out the first-order term in (4.21), thereby enforcing $\delta \tilde{J}^{*}(\eta)=O\left(\eta^{2}\right)$.

5. Numerical case studies. This section computes SS and CS input directions for the optimization of two dynamic systems, namely, a discontinuous chemical reactor in subsection 5.2 and a space shuttle reentry problem in subsection 5.3. The first system is simple as it involves only 2 states, 1 input, and 1 terminal constraint. The second system is more complex as it is highly nonlinear and possesses 5 states, 2 bounded inputs, 1 mixed path constraint, and 3 terminal constraints.

Before presenting the results of the case studies, a numerical procedure that computes the SS and CS components of a parameterized input variation is detailed in subsection 5.1.

5.1. Computing sensitivity- and constraint-seeking directions. The subspaces $\mathcal{V}_{s}^{*}$ and $\mathcal{V}_{c}^{*}$ at the solution of a given optimal control problem are defined implicitly as the kernel of a linear operator and its orthogonal complement, respectively. Moreover, both subspaces can be of infinite dimension; see, e.g., Example 3.1. Determining the projections of an input variation $\left(\boldsymbol{\xi}^{\mathbf{u}}, \boldsymbol{\xi}^{\mathbf{p}}\right) \in \mathcal{V}_{s}^{*}$ in either of these subspaces therefore proves particularly challenging. Instead, we consider approximating these projections using analogous concepts from nonlinear programming (NLP) optimization (see the appendix).

The proposed procedure relies on a direct solution approach $[3,4,16]$, whereby the infinite-dimensional control profiles $\mathbf{u}$ are discretized using a finite number $M \geq 1$ of basis functions $\chi^{i} \in \hat{\mathcal{C}}\left[t_{0}, t_{\mathrm{f}}\right]^{n_{\mathbf{u}}}, i=1, \ldots, M$, so that

$$
\mathbf{u}(t)=\mathcal{U}(t, \mathbf{v}):=\sum_{i=1}^{M} \mathbf{v}^{i} \chi^{i}(t), \quad t \in\left[t_{0}, t_{\mathrm{f}}\right]
$$

The vector of coefficients $\mathbf{v}=\left[\mathbf{v}^{1^{T}} \cdots \mathbf{v}^{M^{T}}\right]^{T} \in \mathbb{R}^{n_{\mathbf{v}}}$, with $n_{\mathbf{v}}=n_{\mathbf{u}} M$, together with the time-invariant parameters $\mathbf{p}$, are the new decision variables in a finite-dimensional 
optimization problem, whose solution approximates that of the original optimal control problem. The path constraints $\boldsymbol{\Omega}$ are also discretized at a finite number of interior points. The resulting discretized optimization problem is an NLP of the form

$$
\begin{aligned}
& \min _{\mathbf{v}, \mathbf{p}} \Phi(\mathbf{v}, \mathbf{p}, \boldsymbol{\theta}) \\
& \text { s.t. } \mathbf{G}(\mathbf{v}, \mathbf{p}, \boldsymbol{\theta}) \leq \mathbf{0} .
\end{aligned}
$$

A numerical procedure that approximates the projections of a given adaptation direction $\left(\boldsymbol{\xi}^{\mathbf{u}}, \boldsymbol{\xi}^{\mathbf{p}}\right)$ on the subspaces $\mathcal{V}_{s}^{*}$ and $\mathcal{V}_{c}^{*}$ is described next. This procedure assumes that the components $\boldsymbol{\xi}^{\mathbf{u}}$ are parameterized in the same way as the inputs $\mathbf{u}$ in (5.1),

$$
\boldsymbol{\xi}^{\mathbf{u}}(t)=\mathcal{U}\left(t, \boldsymbol{\xi}^{\mathbf{v}}\right)=\sum_{i=1}^{M} \boldsymbol{\xi}^{\mathbf{v}^{i}} \chi^{i}(t), \quad t \in\left[t_{0}, t_{\mathrm{f}}\right]
$$

with $\boldsymbol{\xi}^{\mathbf{v}}=\left[\boldsymbol{\xi}^{\mathbf{v}^{1 T}} \cdots \boldsymbol{\xi}^{\mathbf{v}^{M T}}\right]^{T} \in \mathbb{R}^{n_{\mathbf{v}}}$.

For simplicity, we consider piecewise-constant basis functions $\chi^{i}$ on $\left[t_{0}, t_{\mathrm{f}}\right]$ such that the points of discontinuity $t_{i}, i=1, \ldots, M-1$, of $\mathcal{U}(t, \cdot)$ are fixed and evenly spaced on $\left[t_{0}, t_{\mathrm{f}}\right]$,

$$
\mathcal{U}(t, \mathbf{v})=\mathbf{v}^{i} \quad \forall t \in\left[t_{i-1}, t_{i}\right], \quad i=1, \ldots, M .
$$

Moreover, the discretization points for the path constraints $\Omega$ are taken at the same points of discontinuity $t_{i}, i=1, \ldots, M-1$.

Algorithm. Projection of parameterized input variation $\left(\boldsymbol{\xi}^{\mathbf{v}}, \boldsymbol{\xi}^{\mathbf{p}}\right)$ onto SS and CS subspaces.

1. Solve the discretized NLP problem (5.2). Let $\left(\mathbf{v}^{*}, \mathbf{p}^{*}\right)$ be an optimal solution, and let $\mathbf{G}^{a}$ be the vector of active constraints.

2. Apply singular value decomposition (SVD) to the Jacobian matrix $\left[\begin{array}{ll}\mathbf{G}_{\mathbf{v}}^{a} & \mathbf{G}_{\mathbf{p}}^{a}\end{array}\right]$ at $\left(\mathbf{v}^{*}, \mathbf{p}^{*}\right)$ to determine the orthogonal matrices $\mathbf{V}_{s}^{*}$ and $\mathbf{V}_{c}^{*}$ such that

$$
\left[\begin{array}{ll}
\mathbf{G}_{\mathbf{v}}^{a} & \mathbf{G}_{\mathbf{p}}^{a}
\end{array}\right]=\mathbf{U} \boldsymbol{\Sigma}\left[\begin{array}{ll}
\mathbf{V}_{c}^{*} & \mathbf{V}_{s}^{*}
\end{array}\right]^{T} .
$$

(See the appendix for details.)

3. Compute the orthogonal projections of the direction $\left(\boldsymbol{\xi}^{\mathbf{v}}, \boldsymbol{\xi}^{\mathbf{p}}\right)$ on the column spaces of $\mathbf{V}_{s}^{*}$ and $\mathbf{V}_{c}^{*}$,

$$
\left[\begin{array}{l}
\boldsymbol{\xi}_{s}^{\mathbf{v}} \\
\boldsymbol{\xi}_{s}^{\mathbf{p}}
\end{array}\right]=\mathbf{V}_{s}^{*} \mathbf{V}_{s}^{* T}\left[\begin{array}{l}
\boldsymbol{\xi}^{\mathbf{v}} \\
\boldsymbol{\xi}^{\mathbf{p}}
\end{array}\right], \quad \text { and } \quad\left[\begin{array}{l}
\boldsymbol{\xi}_{c}^{\mathbf{v}} \\
\boldsymbol{\xi}_{c}^{\mathbf{p}}
\end{array}\right]=\mathbf{V}_{c}^{*} \mathbf{V}_{c}^{* T}\left[\begin{array}{l}
\boldsymbol{\xi}^{\mathbf{v}} \\
\boldsymbol{\xi}^{\mathbf{p}}
\end{array}\right]
$$

4. Compute the desired input variations $\boldsymbol{\xi}_{s}^{\mathbf{u}}$ and $\boldsymbol{\xi}_{c}^{\mathbf{u}}$ using the parameterization (5.3) as

$$
\boldsymbol{\xi}_{s}^{\mathbf{u}}(t)=\mathcal{U}\left(t, \boldsymbol{\xi}_{s}^{\mathbf{v}}\right), \quad \boldsymbol{\xi}_{c}^{\mathbf{u}}(t)=\mathcal{U}\left(t, \boldsymbol{\xi}_{c}^{\mathbf{v}}\right) \quad \forall t \in\left[t_{0}, t_{\mathrm{f}}\right] .
$$

Steps 3 and 4 of this algorithm are depicted in Figure 2. Given that the projected directions $\left(\boldsymbol{\xi}_{s}^{\mathbf{v}}, \boldsymbol{\xi}_{s}^{\mathbf{p}}\right)$ and $\left(\boldsymbol{\xi}_{c}^{\mathbf{v}}, \boldsymbol{\xi}_{c}^{\mathbf{p}}\right)$ are orthogonal in $\mathbb{R}^{n_{\mathbf{v}}} \times \mathbb{R}^{n_{\mathbf{p}}}$ by construction, and since the parameterization (5.3) is linear in $\boldsymbol{\xi}^{\mathbf{v}}$, it readily follows that the projected directions $\left(\mathcal{U}\left(t, \boldsymbol{\xi}_{s}^{\mathbf{v}}\right), \boldsymbol{\xi}_{s}^{\mathbf{p}}\right)$ and $\left(\mathcal{U}\left(t, \boldsymbol{\xi}_{c}^{\mathbf{v}}\right), \boldsymbol{\xi}_{c}^{\mathbf{p}}\right)$ are themselves orthogonal in $\hat{\mathcal{C}}\left[t_{0}, t_{\mathrm{f}}\right]^{n_{\mathbf{u}}} \times$ $\mathbb{R}^{n_{\mathbf{p}}}$ with respect to the inner product (3.14). It can be shown further that

$$
\mathcal{U}\left(t, \boldsymbol{\xi}^{\mathbf{v}}\right)=\mathcal{U}\left(t, \boldsymbol{\xi}_{s}^{\mathbf{v}}\right)+\mathcal{U}\left(t, \boldsymbol{\xi}_{c}^{\mathbf{v}}\right) \quad \forall t \in\left[t_{0}, t_{\mathrm{f}}\right],
$$

using the property of SVD that $\mathbf{V}_{s}^{*} \mathbf{V}_{s}^{* T}+\mathbf{V}_{c}^{*} \mathbf{V}_{c}^{* T}=\mathbf{I}$. 


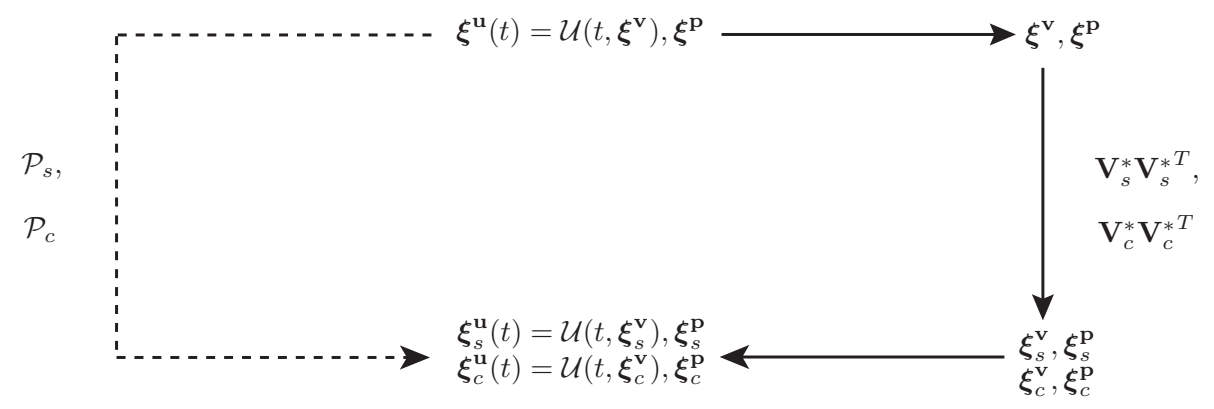

FIG. 2. Computation of $S S$ and $C S$ directions, with exact computations (dotted arrow) and approximate computations (solid arrows). $\mathcal{P}_{s}$ and $\mathcal{P}_{c}$ denote the projection operators onto the subspaces $\mathcal{V}_{s}^{*}$ and $\mathcal{V}_{c}^{*}$, respectively.

Computation of parameterized input variation $\left(\boldsymbol{\xi}^{\mathbf{v}}, \xi^{\mathbf{p}}\right)$. In the case of small parametric perturbations around $\boldsymbol{\theta}^{0}$, a possible - and rather natural - choice of the input adaptation direction is

$$
\left(\boldsymbol{\xi}^{\mathbf{v}}, \boldsymbol{\xi}^{\mathbf{p}}\right)=\left(\mathcal{U}\left(\cdot, \boldsymbol{\xi}^{\mathbf{v}, *}\right), \boldsymbol{\xi}^{\mathbf{p}, *}\right),
$$

where $\left(\boldsymbol{\xi}^{\mathbf{v}, *}, \boldsymbol{\xi}^{\mathbf{p}, *}\right)$ is the (first-order) sensitivity of the nominal optimal solution $\left(\mathbf{v}^{*}, \mathbf{p}^{*}\right)$ of the parametric NLP (5.2) with respect to parameters at $\boldsymbol{\theta}^{0}$; see the appendix.

5.2. Optimization of a batch chemical reactor. This parametric optimal control problem is concerned with the performance optimization of a batch chemical reactor, in which the reversible reaction $A \underset{k_{2}}{\stackrel{k_{1}}{\rightleftharpoons}} B$ takes place nonisothermally [31]. The problem comprises a single input variable, the reactor temperature $u(t)$, and a single (terminal) constraint on the reactant concentration at final time $x_{A}\left(t_{\mathrm{f}}\right)$. The objective is to determine the temperature profile that maximizes the amount of product $B$ for a given batch time. In addition, there is uncertainty in the kinetic parameter $k_{1}$. The optimization problem can be formulated mathematically as

$$
\begin{array}{ll} 
& \max _{u(t)} \quad x_{B}\left(t_{\mathrm{f}}\right) \\
\text { s.t. } & \dot{x}_{A}(t)=-k_{1}(u(t)) x_{A}(t), \quad x_{A}(0)=0.53 \mathrm{kmol} \mathrm{m}^{-3}, \\
& \dot{x}_{B}(t)=k_{1}(u(t)) x_{A}(t)-k_{2}(u(t)) x_{B}(t), \quad x_{B}(0)=0.43 \mathrm{kmol} \mathrm{m}^{-3}, \\
& k_{1}(u(t))=\theta k_{1}^{\circ} \exp \left(-\frac{E_{1}}{u(t)}\right), \quad k_{2}(u(t))=k_{2}^{\circ} \exp \left(-\frac{E_{2}}{u(t)}\right), \\
& x_{A}\left(t_{\mathrm{f}}\right) \leq 0.1 \mathrm{kmol} \mathrm{m}^{-3},
\end{array}
$$

where the parameter $\theta$ denotes the uncertainty in modeling the kinetic parameter $k_{1}$, with nominal value $\theta^{0}=1$. The numerical values of the parameters are given in Table 1. The relative values of $E_{1}$ and $E_{2}$ indicate that low temperatures will push the equilibrium toward the production of $B$, which is desired.

Following the procedure outlined in subsection 5.1, a piecewise-constant input parameterization involving $n=100$ equal-length stages over $\left[0, t_{\mathrm{f}}\right]$ is considered. Figure 3 shows the solution of problem (5.8) reconstructed from the solution $\mathbf{v}^{*}$ of the discretized NLP problem. From an equilibrium point of view, the productivity of $B$ is favored by low temperatures. However, at low temperatures, the reactions proceed 
TABLE 1

Parameter values.

\begin{tabular}{ccl}
\hline Parameter & Value & \\
\hline & & \\
$k_{1}^{\circ}$ & $0.535 \times 10^{11}$ & $\mathrm{~h}^{-1}$ \\
$k_{2}^{\circ}$ & $0.461 \times 10^{18}$ & $\mathrm{~h}^{-1}$ \\
$E_{1}$ & $9 \times 10^{3}$ & $\mathrm{~K}$ \\
$E_{2}$ & $15 \times 10^{3}$ & $\mathrm{~K}$ \\
$t_{\mathrm{f}}$ & 8 & $\mathrm{~h}$ \\
\hline
\end{tabular}
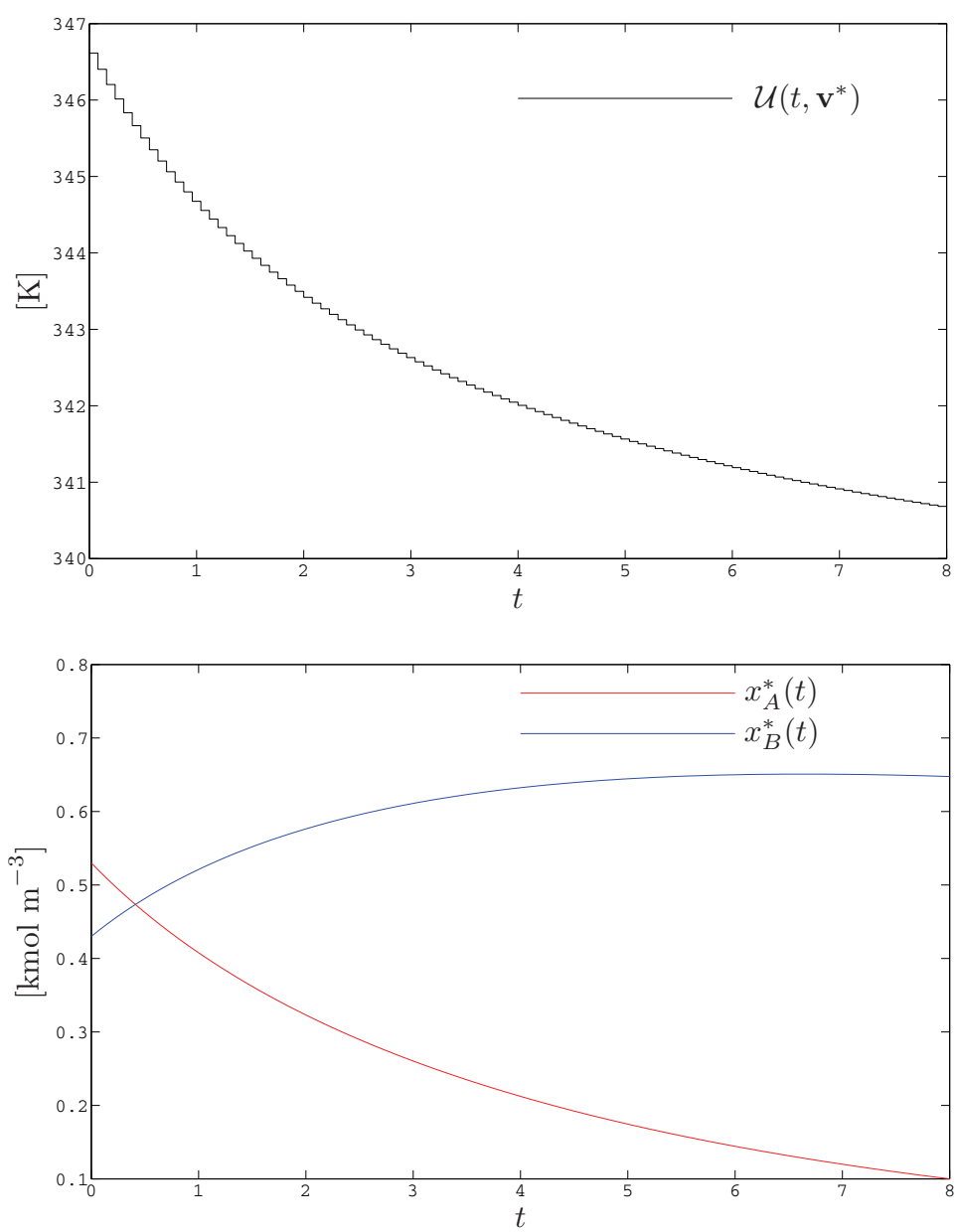

Fig. 3. Nominal optimal temperature profile (top plot) and corresponding concentration trajectories (bottom plot).

slowly and the desired conversion of $A$ will not be achieved in the given batch time. Hence, there exists a compromise, with the temperature being high initially to favor the forward reaction, and reducing with time to limit the backward reaction as more $B$ is produced.

Based on the nominal solution $\mathbf{v}^{*}$ of the NLP problem and on the associated Jacobian $\mathbf{G}_{\mathbf{v}}^{a}$, the projection matrices $\mathbf{V}_{c}^{*} \mathbf{V}_{c}^{* T}$ and $\mathbf{V}_{s}^{*} \mathbf{V}_{s}^{* T}$ are computed according to step 2 of the algorithm in subsection 5.1. The chosen input variation is $\mathcal{U}\left(t, \boldsymbol{\xi}^{\mathbf{v}, *}\right)$, 


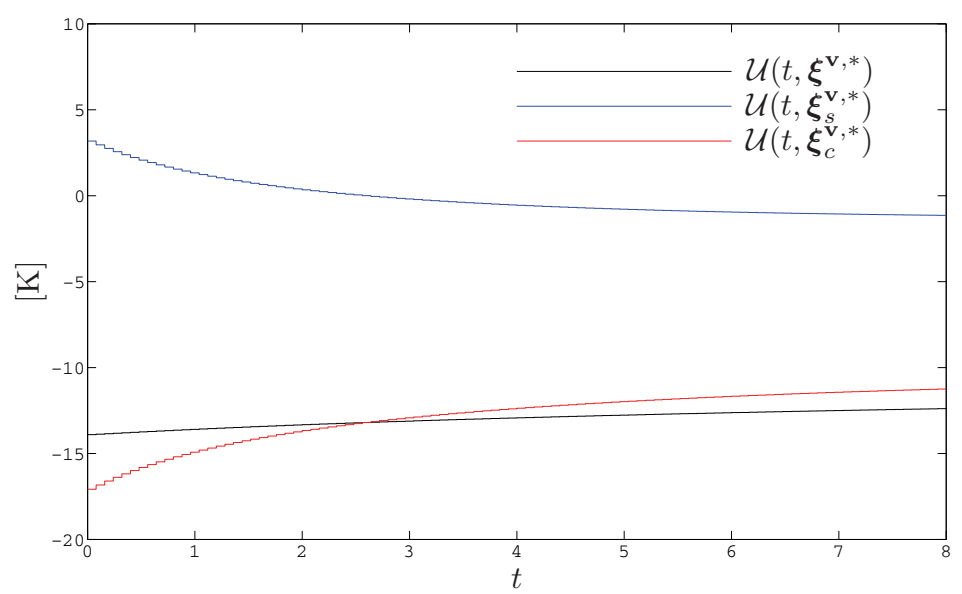

FIG. 4. Input variation $\mathcal{U}\left(t, \boldsymbol{\xi}^{\mathbf{v}, *}\right)$ and its approximated $S S$ and $C S$ components.

as in (5.7). The approximations of its SS and CS components are then computed according to steps 3 and 4 . The input variation profiles are shown in Figure 4. As noted in (5.6), it can be checked that the input variation $\mathcal{U}\left(t, \boldsymbol{\xi}^{\mathbf{v}, *}\right)$ is the pointwise sum of its SS and CS components.

Note that for the considered uncertainty direction $\xi^{\theta}=1$, the forward reaction is faster than in the nominal system when $\eta>0$, which produces more $B$. This allows the optimal temperature to be lower - and thus more favorable from an equilibrium viewpoint - than the nominal optimal solution and still meet the terminal constraint. Figure 4 shows that the input variation $\mathcal{U}\left(t, \boldsymbol{\xi}^{\mathbf{v}, *}\right)$ is negative for all $t$, thus consistent with the need for a lower temperature. The CS component $\mathcal{U}\left(t, \boldsymbol{\xi}_{c}^{\mathbf{v}, *}\right)$ is also negative, which says that, to be able to meet the terminal constraint on $x_{A}\left(t_{\mathrm{f}}\right)$ in the presence of a faster forward reaction, the temperature has to be reduced - and in fact a bit more initially than at the end. In comparison, the SS component $\mathcal{U}\left(t, \boldsymbol{\xi}_{s}^{\mathbf{v}, *}\right)$ is much smaller, initially positive, and then negative, indicating that, for the perturbed reactor, an initially slightly higher temperature followed by a slightly lower temperature would improve productivity without affecting the terminal constraint. Based on the relative magnitudes of the CS and SS components, one might conclude that the input variation in this problem is mostly CS.

Next, parametric perturbations of type (4.1) are considered for $|\eta| \leq 0.05$. The cost variations resulting from input adaptation along the SS direction $\mathcal{U}\left(t, \boldsymbol{\xi}_{s}^{\mathbf{v}, *}\right)$ and along the CS direction $\mathcal{U}\left(t, \boldsymbol{\xi}_{c}^{\mathbf{v}, *}\right)$ are plotted versus $\eta$ in Figure 5. The former can be seen to have an $O\left(\eta^{2}\right)$ fit, while the latter has an $O(\eta)$ fit, which are both consistent with the theory in section 4 .

Note finally that CS adaptation can lead to decreasing the cost value (negative cost variation). For instance, this is the case with $\eta>0$, that is, when the forward reaction is faster than in the nominal case. Indeed, the adaptation forces the terminal constraint to remain active by reducing the temperature, whereas the constraint becomes violated when the nominal solution is applied "as is" to the perturbed system. Note also the relative size of the cost variations resulting from adaptations along the SS and CS directions, with the latter being three orders of magnitude larger than the former. 

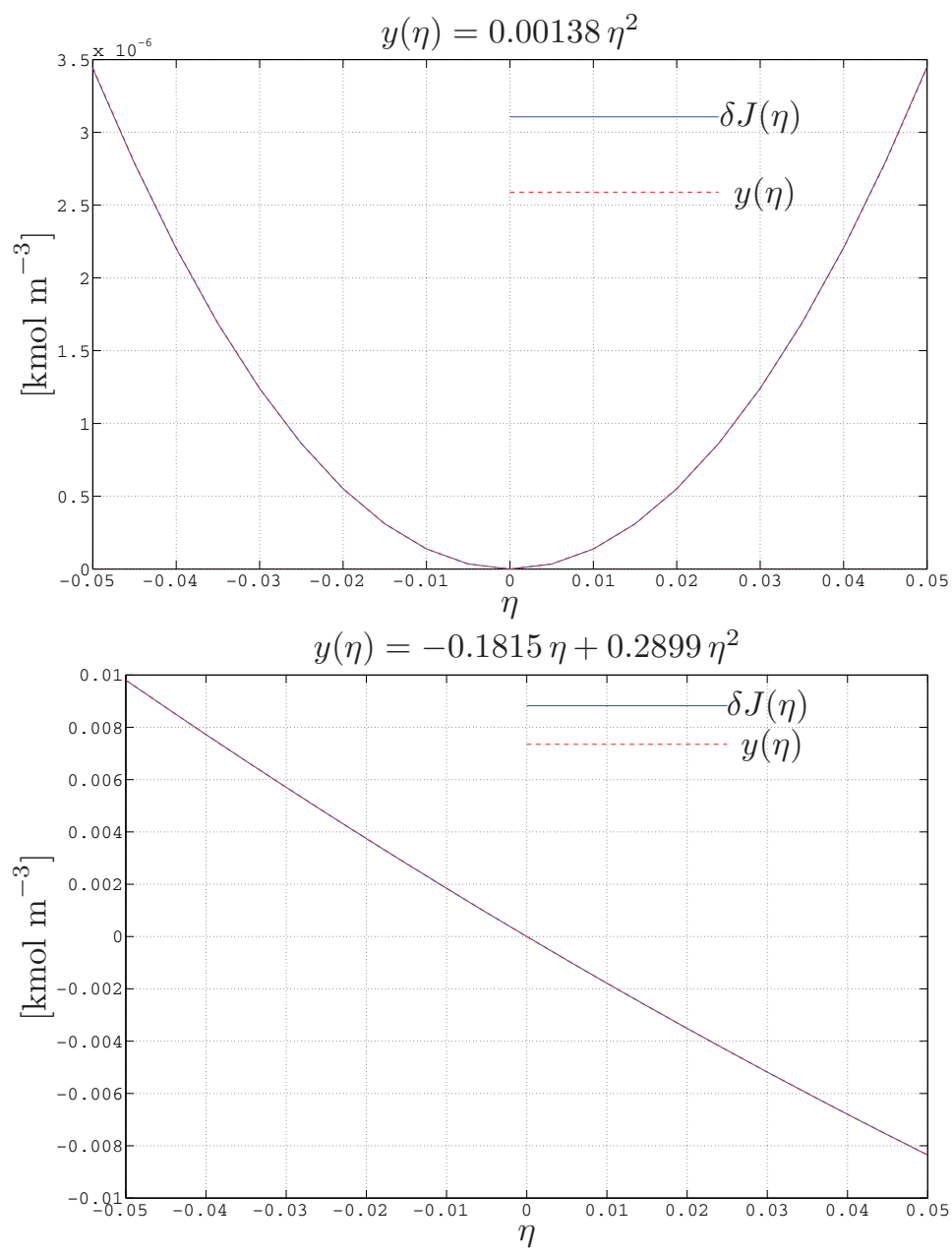

FIG. 5. Cost variation versus $\eta$, resulting from input adaptation along $\mathcal{U}\left(t, \boldsymbol{\xi}_{s}^{\mathbf{v}, *}\right)$ (top plot) and along $\mathcal{U}\left(t, \boldsymbol{\xi}_{c}^{\mathbf{v}, *}\right)$ (bottom plot).

TABLE 2

States and inputs in the space shuttle reentry problem.

\begin{tabular}{llll}
\hline$h$ & altitude (ft) & $a$ & angle of attack (radians) \\
$\vartheta$ & latitude (radians) & $b$ & bank angle (radians) \\
$v$ & velocity (ft / sec) & & \\
$\gamma$ & flight path angle (radians) & & \\
$\psi$ & azimuth (radians) & \\
\hline
\end{tabular}

5.3. Space shuttle reentry trajectory optimization. This subsection investigates directional input adaptation for the optimization of the reentry trajectory of a space shuttle. The parametric optimal control problem is a slight modification of the space shuttle reentry problem in [3] and [17].

The system comprises 5 states and 2 inputs, the details of which are given in Table 2. The optimization problem is subject to lower and upper bounds on both inputs (5.10) and to an upper limit on the aerodynamic heating of the vehicle wing leading 
edge (5.11), which results in a mixed control-state path constraint. Furthermore, there are 3 terminal constraints, namely, an upper limit on the final altitude of the space shuttle and lower limits on its final velocity and final flight path angle (5.12). The objective is to maximize the cross-range of the space shuttle, that is, the final value of its latitude:

$$
\begin{aligned}
\max _{a(t), b(t)} & \vartheta\left(t_{\mathrm{f}}\right) \\
\text { s.t. } & \dot{h}=v \sin \gamma \\
& \dot{\vartheta}=\frac{v}{r} \cos \gamma \cos \psi \\
& \dot{v}=-\frac{D(h, v, a, \theta)}{m}-g(h) \sin \gamma \\
& \dot{\gamma}=\frac{L(h, v, a)}{m v} \cos b+\cos \gamma\left(\frac{v}{r(h)}-\frac{g(h)}{v}\right), \\
& \dot{\psi}=\frac{1}{m v \cos \gamma} L(h, v, a) \sin b+\frac{v}{r \cos \vartheta} \cos \gamma \sin \psi \sin \vartheta \\
& 0 \leq a(t) \leq \frac{\pi}{2} \\
& -\frac{\pi}{2} \leq b(t) \leq-\frac{\pi}{6} \\
& \Omega(a, h, v)=\frac{q_{a}(a) q_{r}(h, v)}{q_{U}}-1.0 \leq 0 \\
& h\left(t_{\mathrm{f}}\right) \leq 8.0 \times 10^{4} \\
& v\left(t_{\mathrm{f}}\right) \geq 2.5 \times 10^{3} \\
& \gamma\left(t_{\mathrm{f}}\right) \geq-\frac{5 \pi}{180}
\end{aligned}
$$

where the functions $r, g, \rho, D, L, q_{a}$, and $q_{r}$ are as follows:

$$
\begin{aligned}
r(h) & =R_{e}+h, \\
g(h) & =\frac{\mu}{r^{2}}, \\
\rho(h) & =\rho_{0} \exp \left(-\frac{h}{h_{r}}\right), \\
D(h, v, a, \theta) & =\frac{1}{2} \theta c_{D} S \rho(h) v^{2}, \quad c_{D}:=a_{0}+a_{1} \hat{a}, \quad \hat{a}:=\frac{180 a}{\pi}, \\
L(h, v, a) & =\frac{1}{2} c_{L} S \rho(h) v^{2}, \quad c_{L}:=b_{0}+b_{1} \hat{a}+b_{2} \hat{a}^{2}, \\
q_{a}(a) & =c_{0}+c_{1} \hat{a}+c_{2} \hat{a}^{2}+c_{3} \hat{a}^{3}, \\
q_{r}(h, v) & =k_{1} \sqrt{\rho}\left(k_{2} v\right)^{3.07} .
\end{aligned}
$$

The parameter $\theta$, with the nominal value $\theta^{0}=1$, is uncertain in the modeling of the aerodynamic drag $D$. The final time $t_{\mathrm{f}}$ is $2000 \mathrm{sec}$. The initial values of the state variables are given in Table 3. All other parameters are specified in Table 4.

The solution of the above problem is obtained by applying a piecewise-constant control vector parameterization involving $n=150$ equidistant stages and discretizing the path constraint at the end-times of each stage. The two nominal optimal inputs and the value of the path constraint are shown in Figure 6. It is found that the 
DIRECTIONAL INPUT ADAPTATION IN OPTIMAL CONTROL

TABLE 3

Initial conditions for the space shuttle reentry problem.

\begin{tabular}{ccccc}
\hline$h(0)$ & $\vartheta(0)$ & $v(0)$ & $\gamma(0)$ & $\psi(0)$ \\
$2.6 \times 10^{5}$ & 0.0 & $2.56 \times 10^{4}$ & $-\pi / 180$ & $\pi / 2$ \\
\hline
\end{tabular}

TABLE 4

Parameter values for the space shuttle reentry problem.

\begin{tabular}{cccc}
\hline Parameter & Value & Parameter & Value \\
\hline$m$ & $6.30944 \times 10^{3}$ & $a_{0}$ & -0.20704 \\
$R_{e}$ & $2.09029 \times 10^{7}$ & $a_{1}$ & $2.9244 \times 10^{-2}$ \\
$\mu$ & $1.4076539 \times 10^{16}$ & $b_{0}$ & $7.854 \times 10^{-2}$ \\
$\rho_{0}$ & $2.378 \times 10^{-3}$ & $b_{1}$ & $-6.1592 \times 10^{-3}$ \\
$h_{r}$ & $2.38 \times 10^{4}$ & $b_{2}$ & $6.21408 \times 10^{-4}$ \\
$q_{U}$ & 70.0 & $c_{0}$ & 1.0672181 \\
$S$ & $2.69 \times 10^{3}$ & $c_{1}$ & $-1.9213774 \times 10^{-2}$ \\
$k_{1}$ & $1.77 \times 10^{4}$ & $c_{2}$ & $2.1286289 \times 10^{-4}$ \\
$k_{2}$ & $1.0 \times 10^{-4}$ & $c_{3}$ & $-1.0117249 \times 10^{-6}$ \\
\hline
\end{tabular}

optimal solution consist of 4 control arcs. Both $a(t)$ and $b(t)$ are in the interior on arc 1 ; then, the path constraint $\Omega$ become binding on $\operatorname{arc} 2 ; a(t)$ and $b(t)$ are again in the interior on arc 3; finally, $b(t)$ is at its upper bound on arc 4 . The nominal state trajectories are depicted in Figure 7. The terminal constraints on the states $v$ and $\gamma$ are seen to be active.

Using the knowledge of the nominal optimal solution, the projection matrices $\mathbf{V}_{c}^{*} \mathbf{V}_{c}^{* T}$ and $\mathbf{V}_{s}^{*} \mathbf{V}_{s}^{* T}$ are computed as described in step 2 of the algorithm in subsection 5.1. The input variations are chosen according to (5.7), and their SS and CS components computed by following steps 3 and 4 are shown in Figure 8. Again, as noted in (5.6), the two input variations $\mathcal{U}\left(t, \boldsymbol{\xi}^{\boldsymbol{\alpha}, *}\right)$ and $\mathcal{U}\left(t, \boldsymbol{\xi}^{\boldsymbol{\beta}, *}\right)$ are equal to the pointwise sum of their respective SS and CS components.

Note that the SS component $\mathcal{U}\left(t, \boldsymbol{\xi}_{s}^{\boldsymbol{\alpha}, *}\right)$ becomes small after about $500 \mathrm{sec}$, an indication that the variation in the input $a(t)$ is mostly CS. The first contribution of $\mathcal{U}\left(t, \boldsymbol{\xi}_{c}^{\boldsymbol{\alpha}, *}\right)$ between $500 \mathrm{sec}$ and $1150 \mathrm{sec}$ is needed to enforce the path constraint; the second contribution, toward the end, is needed to meet a terminal constraint. For the input $b(t)$, since it is at its upper bound after about $1300 \mathrm{sec}$, and since that upper bound does not depend on the parameter $\theta$, the input variation $\mathcal{U}\left(t, \boldsymbol{\xi}^{\boldsymbol{\beta}, *}\right)$ is simply 0 on this arc. Since, except for the initial part, $\mathcal{U}\left(t, \boldsymbol{\xi}_{c}^{\boldsymbol{\beta}, *}\right)$ is small, the variation in the input $b(t)$ is mostly SS.

Finally, parametric perturbations of type (4.1) are considered for $\xi^{\theta}=1$ and $|\eta| \leq 0.05$. The cost variations resulting from input adaptation along the SS direction $\mathcal{U}\left(t, \boldsymbol{\xi}_{s}^{\boldsymbol{\alpha}, *}\right), \mathcal{U}\left(t, \boldsymbol{\xi}_{s}^{\boldsymbol{\beta}, *}\right)$ and along the CS direction $\mathcal{U}\left(t, \boldsymbol{\xi}_{c}^{\boldsymbol{\alpha}, *}\right), \mathcal{U}\left(t, \boldsymbol{\xi}_{c}^{\boldsymbol{\beta}, *}\right)$ are plotted versus $\eta$ in Figure 9. The former (top plot) can be seen to have an $O\left(\eta^{2}\right)$ fit, while the latter (bottom plot) has an $O(\eta)$ fit, which are both consistent with the theory in section 4. Moreover, the former is an order of magnitude smaller than the latter.

6. Conclusions. The complexity of solving optimal control problems plays a decisive role in controller design considerations for practical applications. Various practical limitations dictate that real-time optimization methods should not require recomputing the exact solution. Hence, methods that involve only adaptation of the nominal optimal inputs, which can be computed off-line, at the cost of acceptable optimality loss are appealing. Clearly, a theoretical framework is essential for identifying useful input adaptation schemes and analyzing the effect on the cost of such 

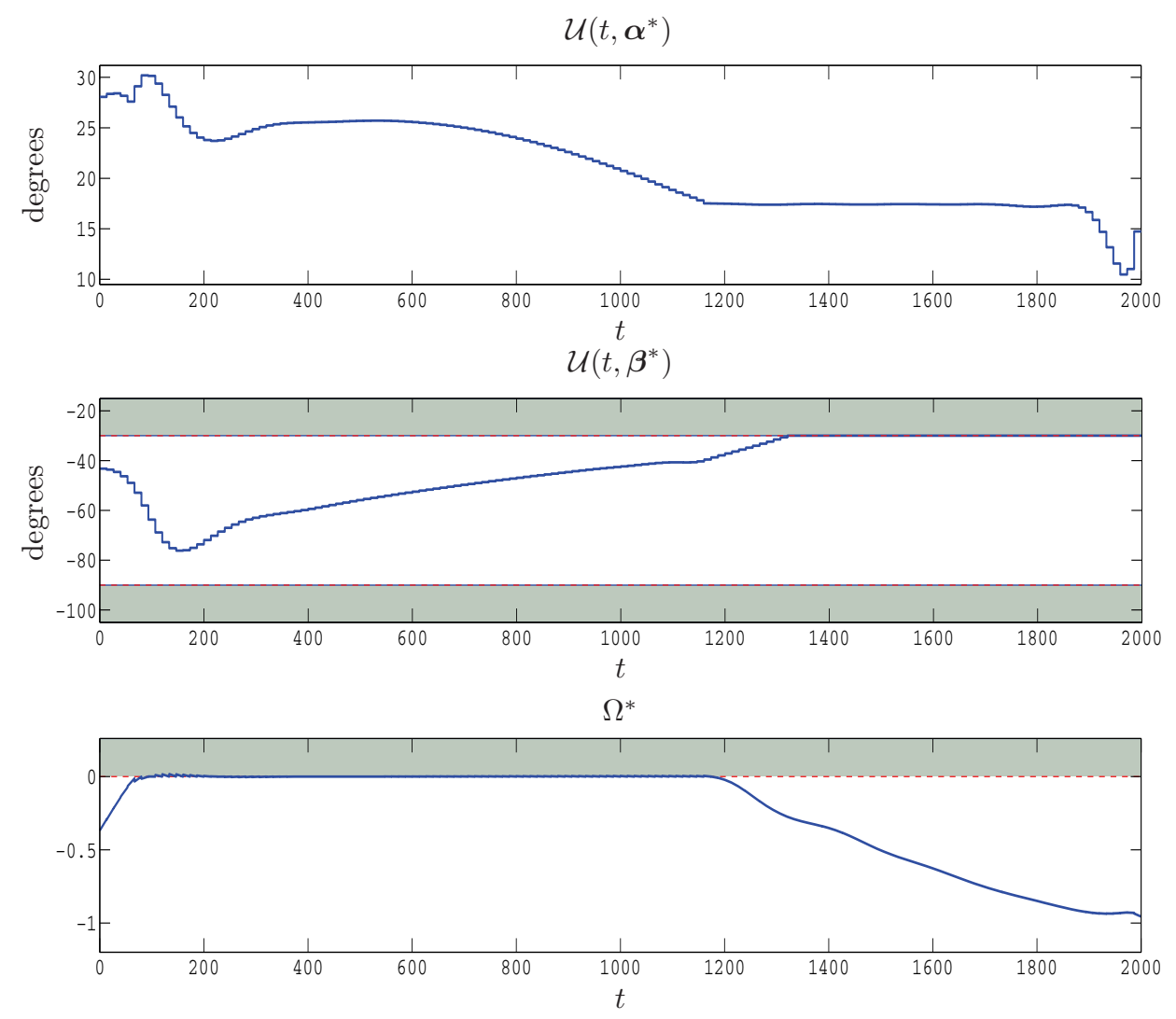

FIG. 6. Two nominal optimal inputs and one path constraint. Dashed lines show the corresponding bounds. Shaded regions depict the regions of infeasible operation.

adaptations. This paper has proposed two input adaptation schemes by focusing on the role of constraints in optimal control problems and analyzing the cost variation that results from each adaptation scheme.

For problems involving terminal and mixed control-state constraints, it is possible to identify directions in the space of input variation functions, along which small variation in the nominal optimal inputs do not cause any change in the active constraints for all $t \in\left[t_{0}, t_{\mathrm{f}}\right]$. These directions are defined as the SS directions and are shown to be solutions of linear integral equations. The directions orthogonal to the set of SS directions are defined as the CS directions.

The main result of the paper is that, in the case of parametric perturbations of type (4.1), the cost variation due to selective input adaptation along SS directionscompared to no input adaptation-is $O\left(\eta^{2}\right)$, whereas it is $O(\eta)$ with selective input adaptation along CS directions. The prime implication of this result for constrained parametric optimal control problems is that, for small parametric perturbations, an adaptation of the inputs along the SS directions has no effect on the first-order term in the optimality loss; only an adaptation along the CS directions can reduce it. Conditions are also derived for a selective input adaptation scheme to cancel out the dominant, first-order term, so that the optimality gap is only $O\left(\eta^{2}\right)$.

These results might prove applicable to a recently developed methodology of 

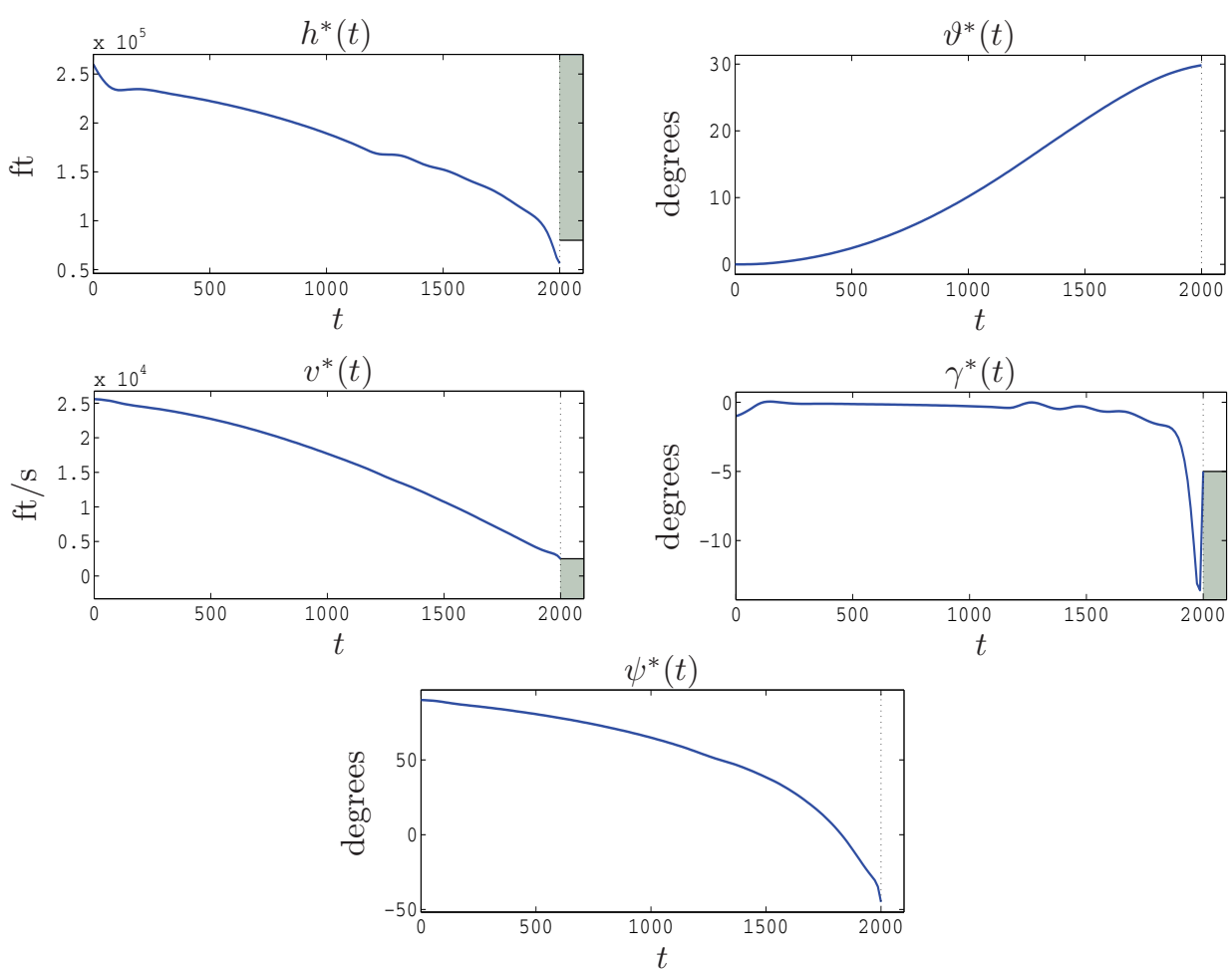

FIG. 7. Five nominal optimal state trajectories. Shaded bands depict the infeasible regions of the respective terminal constraints. The vertical dotted line is the $t=2000$ line.

dynamic real-time optimization for constrained problems called NCO tracking [33, 24], in which the various parts of the input profiles are adapted selectively. Prioritization of selective adaptation strategies is indeed crucial for the implementation of practical NCO-tracking controllers. The analysis in this paper also provides a sound theoretical basis for constraint adaptation schemes such as [27] and could help further improve existing on-line adaptation strategies such as $[11,37,36]$.

The results in sections 3 and 4 were obtained under the assumption of continuous optimal controls $\mathbf{u}^{*}$. However, this is not the case for many practical applications. Fortunately, careful inspection of the derivations reveals that the procedure is not limited by the requirement of continuity of $\mathbf{u}^{*}$, although the developments are greatly simplified by the latter assumption. Indeed, if we include the points of discontinuity of $\mathbf{u}^{*}$ in the set $\mathcal{T}$, a similar derivation holds provided that the number of continuous arcs in $\mathbf{u}^{*}$ remains finite. Furthermore, since the results are valid for piecewise-continuous $\mathbf{u}^{*}$, we can forgo the assumption of regularity of the Hamiltonian. This should allow the results to be extended to more complex problems such as singular optimal control problems.

Finally, in the case of problems having pure state constraints, additional care must be taken in the derivation since there might be jumps in the adjoint functions $\boldsymbol{\lambda}^{*}$ at some interior points $[20,28]$. Since the nominal solution is known, these interior points are known and need to be added to $\mathcal{T}$. Definition 3.2 of the SS directions would include an additional condition - in the form of suitable integral equations - representing the zero change in the values of the pure state constraints due to small local variation in 

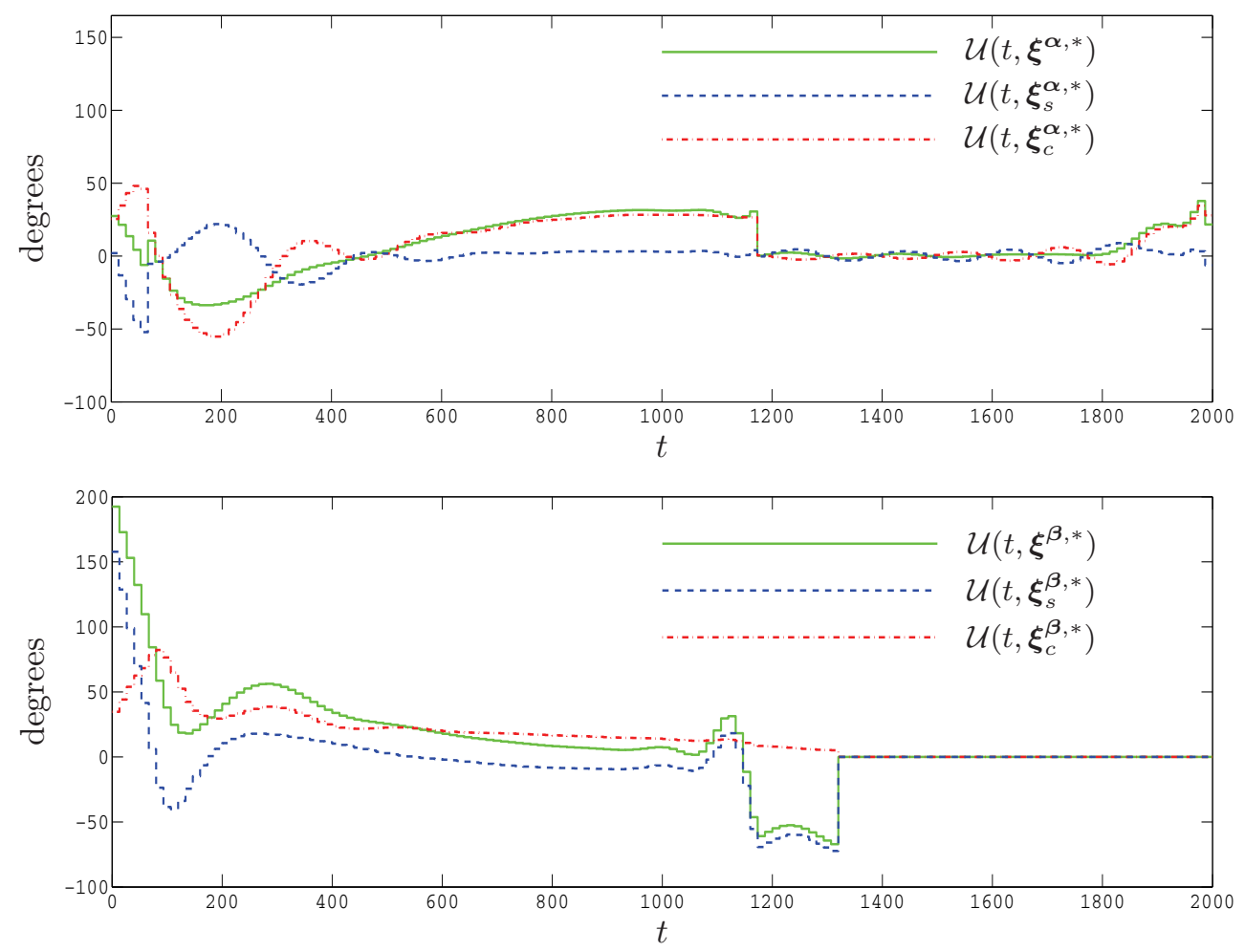

FIG. 8. SS and $C S$ components of $\mathcal{U}\left(t, \boldsymbol{\alpha}^{*}\right)$ and $\mathcal{U}\left(t, \boldsymbol{\beta}^{*}\right)$.

the nominal inputs of type (3.1), (3.2). In the cost variational analysis, the expression of $\boldsymbol{\beta}^{*}$ in (4.12), and thus also that of $\delta J(\eta)$ in (4.13) and (4.14), would be modified to accommodate the appropriate terms involving the pure state constraints. The rest of the procedure, and thus also the results, would remain essentially the same.

Appendix. Selective input adaptation in NLP problems. This appendix is a digest of results in selective adaptation of optimal control problems governed by stationary processes $[15,9]$.

Consider a parametric NLP problem of the form

$(\operatorname{NLP}(\boldsymbol{\theta}))$

$$
\begin{aligned}
& \min _{\mathbf{p}} \Phi(\mathbf{p}, \boldsymbol{\theta}) \\
& \text { s.t. } \mathbf{G}(\mathbf{p}, \boldsymbol{\theta}) \leq \mathbf{0},
\end{aligned}
$$

where $\mathbf{p} \in \mathbb{R}^{n_{\mathbf{p}}}$ and $\boldsymbol{\theta} \in \Theta \subset \mathbb{R}^{n_{\boldsymbol{\theta}}}$. Assume that an optimal solution $\mathbf{p}^{*}$ of the NLP problem is known at the nominal value $\boldsymbol{\theta}^{0}$ of the uncertain parameters. Assume further that the following linear independence constraint qualification (LICQ) holds at the optimal nominal solution,

$$
\operatorname{rank}\left[\mathbf{G}_{\mathbf{p}}\left(\mathbf{p}^{*}, \boldsymbol{\theta}^{0}\right) \operatorname{diag}\left(\mathbf{G}\left(\mathbf{p}^{*}, \boldsymbol{\theta}^{0}\right)\right)\right]=n_{\mathbf{G}}^{a},
$$

with $n_{\mathbf{G}}^{a}$ the number of active constraints at $\mathbf{p}^{*}$. Then, provided that $\Phi$ and $\mathbf{G}$ are differentiable at $\mathbf{p}^{*}$, the following NCOs must hold [2],

$$
\begin{aligned}
& \mathbf{G}\left(\mathbf{p}^{*}, \boldsymbol{\theta}^{0}\right) \leq \mathbf{0}, \quad \boldsymbol{\nu}^{* T} \mathbf{G}\left(\mathbf{p}^{*}, \boldsymbol{\theta}^{0}\right)=0, \quad \boldsymbol{\nu}^{*} \geq \mathbf{0}, \\
& \Phi_{\mathbf{p}}\left(\mathbf{p}^{*}, \boldsymbol{\theta}^{0}\right)+\boldsymbol{\nu}^{* T} \mathbf{G}_{\mathbf{p}}\left(\mathbf{p}^{*}, \boldsymbol{\theta}^{0}\right)=\mathbf{0},
\end{aligned}
$$



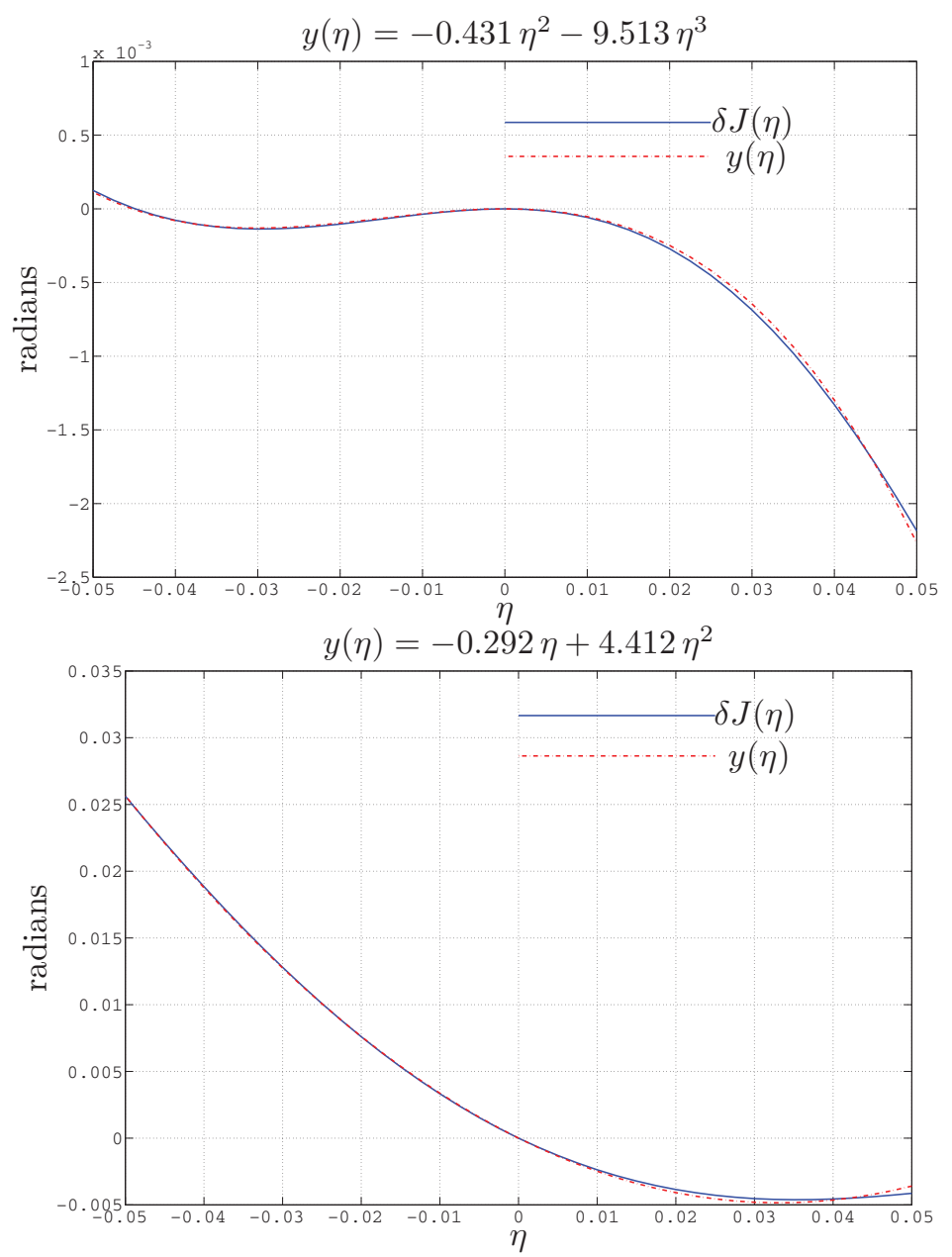

FIG. 9. Cost variation versus $\eta$, resulting from input adaptation along $\mathcal{U}\left(t, \boldsymbol{\xi}_{s}^{\boldsymbol{\alpha}, *}\right), \mathcal{U}\left(t, \boldsymbol{\xi}_{s}^{\boldsymbol{\beta}, *}\right)$ (top plot) and along $\mathcal{U}\left(t, \boldsymbol{\xi}_{c}^{\boldsymbol{\alpha} *}\right), \mathcal{U}\left(t, \boldsymbol{\xi}_{c}^{\boldsymbol{\beta}, *}\right.$ ) (bottom plot).

for a (unique) Lagrange multiplier vector $\boldsymbol{\nu}^{*} \in \mathbb{R}^{n_{\mathbf{G}}}$.

Since the number of active constraints $n_{\mathbf{G}}^{a}$ at $\mathbf{p}^{*}$ is less than $n_{\mathbf{p}}$, there exist directions in the input parameter space along which taking an infinitesimal step from an optimal solution point does not modify the active constraints. Subsequently, the active constraints are denoted by $\mathbf{G}^{a}$, and the corresponding Lagrange multiplier vector are denoted by $\boldsymbol{\nu}^{a} \in \mathbb{R}^{n_{\mathrm{G}}^{a}}$.

DeFINITION A.1 (CS and SS directions in NLP problems). The direction $\boldsymbol{\xi}^{\mathbf{p}} \in \mathbb{R}^{n_{\mathbf{p}}}$ is called an SS direction for the optimization problem $\operatorname{NLP}\left(\boldsymbol{\theta}^{0}\right)$ at $\mathbf{p}^{*}$ if $\boldsymbol{\xi}^{\mathbf{p}} \in \operatorname{ker}\left(\mathbf{G}_{\mathbf{p}}^{a}\left(\mathbf{p}^{*}, \boldsymbol{\theta}^{0}\right)\right)$. $\boldsymbol{\xi}^{\mathbf{p}}$ is called a CS direction for $\operatorname{NLP}\left(\boldsymbol{\theta}^{0}\right)$ at $\mathbf{p}^{*}$ if $\boldsymbol{\xi}^{\mathbf{p}} \in$ $\operatorname{ker}\left(\mathbf{G}_{\mathbf{p}}^{a}\left(\mathbf{p}^{*}, \boldsymbol{\theta}^{0}\right)\right)^{\perp}:=\left\{\boldsymbol{\zeta} \in \mathbb{R}^{n_{\mathbf{p}}}: \boldsymbol{\zeta}^{T} \boldsymbol{\xi}=0\right.$ for all $\left.\boldsymbol{\xi} \in \operatorname{ker}\left(\mathbf{G}_{\mathbf{p}}^{a}\left(\mathbf{p}^{*}, \boldsymbol{\theta}^{0}\right)\right)\right\}$.

Clearly, an infinitesimal step along a CS direction away from $\mathbf{p}^{*}$ modifies the active constraint values, whereas an infinitesimal step along a SS direction leaves the active constraints unchanged. 
If LICQ holds at $\mathbf{p}^{*}$, the Jacobian matrix $\mathbf{G}_{\mathbf{p}}^{a}\left(\mathbf{p}^{*}, \boldsymbol{\theta}^{0}\right)$ is full rank and SVD gives

$$
\mathbf{G}_{\mathbf{p}}^{a}\left(\mathbf{p}^{*}, \boldsymbol{\theta}^{0}\right)=\mathbf{U} \boldsymbol{\Sigma} \mathbf{V}^{T}
$$

where $\boldsymbol{\Sigma}$ is an $\left(n_{\mathbf{G}}^{a} \times n_{\mathbf{p}}\right)$ matrix of the form

$$
\boldsymbol{\Sigma}=\left[\begin{array}{cccccc}
\sigma_{1} & & & 0 & \cdots & 0 \\
& \ddots & & \vdots & \ddots & \vdots \\
& & \sigma_{n_{\mathrm{G}}^{a}} & 0 & \cdots & 0
\end{array}\right]:=\left[\begin{array}{ll}
\boldsymbol{\Sigma}_{c} & 0
\end{array}\right],
$$

$\mathbf{U}$ is an $\left(n_{\mathbf{G}}^{a} \times n_{\mathbf{G}}^{a}\right)$ orthonormal matrix, and $\mathbf{V}:=\left[\mathbf{V}_{c}^{*} \mathbf{V}_{s}^{*}\right]$ is an $\left(n_{\mathbf{p}} \times n_{\mathbf{p}}\right)$ orthonormal matrix. The $n_{\mathbf{G}}^{a}$ columns of $\mathbf{V}_{c}^{*}$ are a basis of the $C S$ subspace at $\mathbf{p}^{*}$, while the $\left(n_{\mathbf{p}}-n_{\mathbf{G}}^{a}\right)$ columns of $\mathbf{V}_{s}^{*}$ are a basis of the SS subspace at $\mathbf{p}^{*}$.

Now, consider a variation of the parameters from their nominal values $\boldsymbol{\theta}^{0}$ as $\tilde{\boldsymbol{\theta}}(\eta)=\boldsymbol{\theta}^{0}+\eta \boldsymbol{\xi}^{\boldsymbol{\theta}}$, with $\boldsymbol{\xi}^{\boldsymbol{\theta}} \in \mathbb{R}^{n_{\boldsymbol{\theta}}}$ and $|\eta| \ll 1$. Let $\boldsymbol{\xi}^{\mathbf{p}} \in \mathbb{R}^{n_{\mathbf{p}}}$ be a direction of adaptation in the optimization variable space, so that $\tilde{\mathbf{p}}(\eta)=\mathbf{p}^{*}+\eta \boldsymbol{\xi}^{\mathbf{p}}$. Clearly, $\boldsymbol{\xi}^{\mathbf{p}}$ can be (uniquely) decomposed as $\boldsymbol{\xi}^{\mathbf{p}}=\boldsymbol{\xi}_{c}^{\mathbf{p}}+\boldsymbol{\xi}_{s}^{\mathbf{p}}$, where $\boldsymbol{\xi}_{c}^{\mathbf{p}}:=\mathbf{V}_{c}^{*} \mathbf{V}_{c}^{* T} \boldsymbol{\xi}^{\mathbf{p}}$ and $\boldsymbol{\xi}_{s}^{\mathbf{p}}:=\mathbf{V}_{s}^{*} \mathbf{V}_{s}^{* T} \boldsymbol{\xi}^{\mathbf{p}}$ are CS and SS directions, respectively.

The cost variation $\delta \Phi$ upon input adaptation in the direction $\boldsymbol{\xi}^{\mathbf{p}}$ is given by

$$
\delta \Phi(\eta)=\Phi\left(\mathbf{p}^{*}+\eta \boldsymbol{\xi}^{\mathbf{p}}, \tilde{\boldsymbol{\theta}}(\eta)\right)-\Phi\left(\mathbf{p}^{*}, \tilde{\boldsymbol{\theta}}(\eta)\right) .
$$

Approximation orders for this variation are derived in the following theorem for selective input adaptation along the CS and SS directions.

ThEOREM A.2 (cost variation resulting from selective input adaptation in NLP problems). Let $\mathbf{u}^{*}$ be an optimal solution of $\operatorname{NLP}\left(\boldsymbol{\theta}^{0}\right)$, and consider the parametric variations $\tilde{\boldsymbol{\theta}}(\eta)=\boldsymbol{\theta}^{0}+\eta \boldsymbol{\xi}^{\boldsymbol{\theta}}$. The cost variation $\delta \Phi(\eta)$ resulting from the selective input adaptation $\tilde{\mathbf{p}}(\eta)=\mathbf{p}^{*}+\eta \boldsymbol{\xi}^{\mathbf{p}}$ is $O(\eta)$ along a $C S$ direction and is $O\left(\eta^{2}\right)$ along a $S S$ direction.

Proof. The cost variation $\delta \Phi(\eta)$ in (A.3) can be expressed as

$$
\delta \Phi(\eta)=\eta \Phi_{\mathbf{p}}\left(\mathbf{p}^{*}, \tilde{\boldsymbol{\theta}}(\eta)\right) \boldsymbol{\xi}^{\mathbf{p}}+O\left(\eta^{2}\right)=\eta \Phi_{\mathbf{p}}\left(\mathbf{p}^{*}, \boldsymbol{\theta}^{0}\right) \boldsymbol{\xi}^{\mathbf{p}}+O\left(\eta^{2}\right) .
$$

From the NCOs (A.1), (A.2) at $\mathbf{p}^{*}$, we have

$$
\delta \Phi(\eta)=-\eta \boldsymbol{\nu}^{* T} \mathbf{G}_{\mathbf{p}}\left(\mathbf{p}^{*}, \boldsymbol{\theta}^{0}\right) \boldsymbol{\xi}^{\mathbf{p}}+O\left(\eta^{2}\right)=-\eta \boldsymbol{\nu}^{a T} \mathbf{G}_{\mathbf{p}}^{a}\left(\mathbf{p}^{*}, \boldsymbol{\theta}^{0}\right) \boldsymbol{\xi}^{\mathbf{p}}+O\left(\eta^{2}\right) .
$$

Since any SS direction $\boldsymbol{\xi}^{\mathbf{p}}$ is in $\operatorname{ker}\left(\mathbf{G}_{\mathbf{p}}^{a}\left(\mathbf{p}^{*}, \boldsymbol{\theta}^{0}\right)\right)$, it follows that $\delta \Phi(\eta)$ is $O\left(\eta^{2}\right)$ upon selective adaptation along such directions. On the other hand, $\delta \Phi(\eta)$ is $O(\eta)$ upon selective adaptation along CS directions.

In practice, a natural choice for the direction of input adaptation $\xi^{\mathbf{p}}$ is the sensitivity of the optimal nominal solution $\mathbf{p}^{*}$ with respect to the parameters at $\boldsymbol{\theta}^{0}$. With a small abuse of notation, let $\mathbf{p}^{*}(\eta)$ denote the solutions of $\operatorname{NLP}(\tilde{\boldsymbol{\theta}}(\eta))$ for small parametric solutions $|\eta| \ll 1$, and let $\boldsymbol{\nu}^{*}(\eta)$ denote the corresponding Lagrange multiplier vectors; we thus have $\mathbf{p}^{*}(0)=\mathbf{p}^{*}$ and $\boldsymbol{\nu}^{*}(0)=\boldsymbol{\nu}^{*}$. Under the additional assumptions that

i. $\Phi$ and $\mathbf{G}$ are twice-continuously differentiable with respect to $\mathbf{p}$ and $\boldsymbol{\theta}$ at $\mathbf{p}^{*}, \boldsymbol{\theta}^{0}$

ii. the following second-order sufficient condition holds at $\mathbf{p}^{*}$ :

$$
\boldsymbol{\xi}^{T}\left(\Phi_{\mathbf{p p}}\left(\mathbf{p}^{*}, \boldsymbol{\theta}^{0}\right)+\boldsymbol{\nu}^{* T} \mathbf{G}_{\mathbf{p p}}\left(\mathbf{p}^{*}, \boldsymbol{\theta}^{0}\right)\right) \boldsymbol{\xi}>0 \quad \forall \boldsymbol{\xi} \in \operatorname{ker}\left(\mathbf{G}_{\mathbf{p}}^{a}\left(\mathbf{p}^{*}, \boldsymbol{\theta}^{0}\right)\right) ;
$$


iii. strict complementarity slackness $\boldsymbol{\nu}^{a}>\mathbf{0}$ holds at $\mathbf{p}^{*}$, the vector functions $\mathbf{p}^{*}$ and $\boldsymbol{\nu}^{*}$ are guaranteed to exist and be continuously differentiable with respect to $\eta$ in a neighborhood of $\eta=0$ [14], and the active set remains unchanged in that neighborhood. Moreover, the optimum point and Lagrange multiplier sensitivities can be computed as

$$
\left[\begin{array}{c}
\boldsymbol{\xi}^{\mathbf{p}, *} \\
\boldsymbol{\xi}^{\boldsymbol{\nu}, *}
\end{array}\right]=-\left[\begin{array}{cc}
\Phi_{\mathbf{p p}}+\boldsymbol{\nu}^{* T} \mathbf{G}_{\mathbf{p p}} & \mathbf{G}_{\mathbf{p}}^{T} \\
\operatorname{diag}\left(\mathbf{G}_{\mathbf{p}}\right) \boldsymbol{\nu}^{*} & \operatorname{diag}(\mathbf{G})
\end{array}\right]_{\mathbf{p}^{*}, \boldsymbol{\theta}^{0}}^{-1}\left[\begin{array}{c}
\Phi_{\mathbf{p} \boldsymbol{\theta}}+\boldsymbol{\nu}^{* T} \mathbf{G}_{\mathbf{p} \boldsymbol{\theta}} \\
\operatorname{diag}\left(\mathbf{G}_{\boldsymbol{\theta}}\right) \boldsymbol{\nu}^{*}
\end{array}\right]_{\mathbf{p}^{*}, \boldsymbol{\theta}^{0}} \boldsymbol{\xi}^{\boldsymbol{\theta}} .
$$

Let $\delta \Phi^{\star}(\eta)$ denote the actual optimality loss due to selective adaptation in the direction $\boldsymbol{\xi}^{\mathbf{p}}$, as compared to an adaptation in the optimal direction $\boldsymbol{\xi}^{\mathbf{p}, *}$,

$$
\delta \Phi^{\star}(\eta)=\Phi\left(\mathbf{p}^{*}+\eta \boldsymbol{\xi}^{\mathbf{p}, *}, \tilde{\boldsymbol{\theta}}(\eta)\right)-\Phi\left(\mathbf{p}^{*}+\eta \boldsymbol{\xi}^{\mathbf{p}}, \tilde{\boldsymbol{\theta}}(\eta)\right) .
$$

It can be established that, for $|\eta| \ll 1, \delta \Phi^{\star}(\eta)$ is such that

$$
\delta \Phi^{\star}(\eta)=\eta \boldsymbol{\nu}^{a T} \mathbf{G}_{\boldsymbol{\theta}}^{a}\left(\mathbf{p}^{*}, \boldsymbol{\theta}^{0}\right) \boldsymbol{\xi}^{\boldsymbol{\theta}}+O\left(\eta^{2}\right) .
$$

Therefore, it follows by Theorem A.2 that no input adaptation scheme along an SS direction is able to close the dominant term $\eta \boldsymbol{\nu}^{a T} \mathbf{G}_{\boldsymbol{\theta}}^{a}\left(\mathbf{p}^{*}, \boldsymbol{\theta}^{0}\right) \boldsymbol{\xi}^{\boldsymbol{\theta}}$ in this optimality gap; only adaptation along a CS direction is able to reduce such an optimality gap. In particular, any adaptation direction $\boldsymbol{\xi}^{\mathbf{p}}$ such that

$$
\mathbf{G}_{\mathbf{p}}^{a}\left(\mathbf{p}^{*}, \boldsymbol{\theta}^{0}\right) \xi^{\mathbf{p}}+\mathbf{G}_{\boldsymbol{\theta}}^{a}\left(\mathbf{p}^{*}, \boldsymbol{\theta}^{0}\right) \boldsymbol{\xi}^{\boldsymbol{\theta}}=\mathbf{0}
$$

will cancel out the dominant, first-order term in (A.4) and enforce $\delta \Phi^{\star}(\eta)=O\left(\eta^{2}\right)$.

\section{REFERENCES}

[1] M. AnItescu, Spectral finite-element methods for parametric constrained optimization problems, SIAM J. Numer. Anal., 47 (2009), pp. 1739-1759.

[2] M. S. Bazaraa, H. D. Sherali, and C. M. Shetty, Nonlinear Programming: Theory and Algorithms, 2nd ed., John Wiley \& Sons, New York, 1993.

[3] J. T. Betts, Practical Methods for Optimal Control and Estimation Using Nonlinear Programming, 2nd ed., Adv. Des. Control 19, SIAM, Philadelphia, 2010.

[4] L. T. Biegler, Nonlinear Programming: Concepts, Algorithms, and Applications to Chemical Processes, MOS-SIAM Ser. Optim. 10, SIAM, Philadelphia, 2010.

[5] A. E. Bryson and Y. C. Ho, Applied Optimal Control, Hemisphere, Washington, DC, 1975.

[6] C. Büskens ANd H. Maurer, Sensitivity analysis and real-time control of parametric optimal control problems using nonlinear programming methods, in Online Optimization of Large Scale Systems, M. Grötschel, S. O. Krumke, and J. Rambau, eds., Springer-Verlag, Berlin, 2001, pp. 57-68.

[7] C. BÜSKens And H. MaUrer, Sensitivity analysis and real-time optimization of parametric nonlinear programming problems, in Online Optimization of Large Scale Systems, M. Grötschel, S. O. Krumke, and J. Rambau, eds., Springer-Verlag, Berlin, 2001, pp. 3-16.

[8] L. Cesari, Optimization: Theory and Applications, Appl. Math., Springer, New York, 1983.

[9] B. Chachuat, A. Marchetti, and D. Bonvin, Process optimization via constraints adaptation, J. Process Control, 18 (2008), pp. 244-257.

[10] S. Deshpande, B. Chachuat, and D. Bonvin, Parametric sensitivity of path-constrained optimal control: Towards selective input adaptation, in Proceedings of the American Control Conference, IEEE Press, Piscataway, NJ, 2009, pp. 349-354.

[11] M. Dienl, H. G. Bock, AND J. P. SchlÖder, A real-time iteration scheme for nonlinear optimization in optimal feedback control, SIAM J. Control Optim., 43 (2005), pp. 17141736. 
[12] M. Diehl, H. G. Bock, J. P. Schlöder, R. Findeisen, Z. NAGy, and F. Allgöwer, Real-time optimization and nonlinear model predictive control of processes governed by differentialalgebraic equations, J. Process Control, 12 (2002), pp. 577-585.

[13] S. Dineen, Multivariate Calculus and Geometry, 2nd ed., Springer, London, UK, 2001.

[14] A. V. FIACCO, Introduction to Sensitivity and Stability Analysis in Nonlinear Programming, Math. Sci. Eng. 165, Academic Press, New York, 1983.

[15] G. François, B. SRinivasan, And D. Bonvin, Use of measurements for enforcing the necessary conditions of optimality in the presence of constraints and uncertainty, J. Process Control, 15 (2005), pp. 701-712.

[16] C. GoH AND K. Teo, Control parameterization: A unified approach to optimal control problems with general constraints, Automatica J. IFAC, 24 (1988), pp. 3-18.

[17] K. Graichen And N. Petit, Constructive methods for initialization and handling mixed stateinput constraints in optimal control, J. Guidance Control Dynam., 31 (2008), pp. 13341343.

[18] S. Gros, B. Chachuat, and D. Bonvin, Neighbouring-extremal control for singular dynamic optimisation problems. II. Multiple-input case, Int. J. Control, 82 (2009), pp. 1193-1211.

[19] S. Gros, B. Srinivasan, B. Chachuat, and D. Bonvin, Neighbouring-extremal control for singular dynamic optimization problems. I. Single-input system, Int. J. Control, 82 (2009), pp. 1099-1112.

[20] R. F. Hartl, S. P. Sethi, and R. G. Vickson, A survey of the maximum principles for optimal control problems with state constraints, SIAM Rev., 37 (1995), pp. 181-218.

[21] K. Ito AND K. KunISCH, Sensitivity analysis of solutions to optimization problems in Hilbert spaces with applications to optimal control and estimation, J. Differential Equations, 99 (1992), pp. 1-40.

[22] K. Ito And K. Kunisch, Lagrange Multiplier Approach to Variational Problems and Applications, Adv. Des. Control 15, SIAM, Philadelphia, 2008.

[23] J. KadAm AND W. MarquardT, Integration of economical optimization and control for intentionally transient process operation, in Assessment and Future Directions of Nonlinear Model Predictive Control, R. Findeisen, F. Allgöwer, and L. T. Biegler, eds., Springer, Berlin, 2007, pp. 419-434.

[24] J. Kadam, M. Schlegel, B. Srinivasan, D. Bonvin, and W. Marquardt, Dynamic optimization in the presence of uncertainty: From off-line nominal solution to measurementbased implementation, J. Process Control, 17 (2007), pp. 389-398.

[25] J. R. Magnus And H. Neudecker, Matrix Differential Calculus with Applications in Statistics and Econometrics, John Wiley \& Sons, Inc., Chichester, 1988.

[26] K. Malanowski and H. Maurer, Sensitivity analysis for parametric control problems with control-state constraints, Comput. Optim. Appl., 5 (1996), pp. 253-283.

[27] A. Marchetti, B. Chachuat, and D. Bonvin, Batch process optimization via run-to-run constraints adaptation, in Proceedings of the European Control Conference (Kos, Greece), 2007.

[28] H. Maurer And D. Augustin, Sensitivity analysis and real-time control of parametric optimal control problems using boundary value methods, in Online Optimization of Large Scale Systems, M. Grötschel, S. O. Krumke, and J. Rambau, eds., Springer-Verlag, Berlin, 2001, pp. $17-55$.

[29] H. Maurer And H. J. Pesch, Solution differentiability for parametric nonlinear control problems with control-state constraints, J. Optim. Theory Appl., 86 (1995), pp. 285-309.

[30] H. J. PESCH, Real-time computation of feedback controls for constrained optimal control problems. I. Neighbouring extremals, Optimal Control Appl. Methods, 10 (1989), pp. 129-145.

[31] W. F. Ramirez, Process Control and Identification, Academic Press, New York, 1994.

[32] W. J. Rugh, Linear System Theory, Prentice Hall, Englewood Cliffs, NJ, 1993.

[33] B. SRinivasan and D. Bonvin, Real-time optimization of batch processes via tracking of necessary conditions of optimality, Ind. Eng. Chem. Res., 46 (2007), pp. 492-504.

[34] K. L. Teo, C. J. GoH, And K. H. Wong, A Unified Computational Approach to Optimal Control Problems, Pitman Monogr. Surveys Pure Appl. Math. 55, John Wiley \& Sons, New York, 1991.

[35] A. Wouk, A Course of Applied Functional Analysis, John Wiley \& Sons, Inc., New York, 1979.

[36] L. Würth, R. Hannemann, and W. Marquardt, A two-layer architecture for economically optimal process control and operation, J. Process Control, 21 (2011), pp. 311-321.

[37] V. M. Zavala, C. Laird, And L. Biegler, Fast implementations and rigorous models: Can both be accommodated in NMPC?, Int. J. Robust Nonlinear Control, 18 (2008), pp. 800815 . 